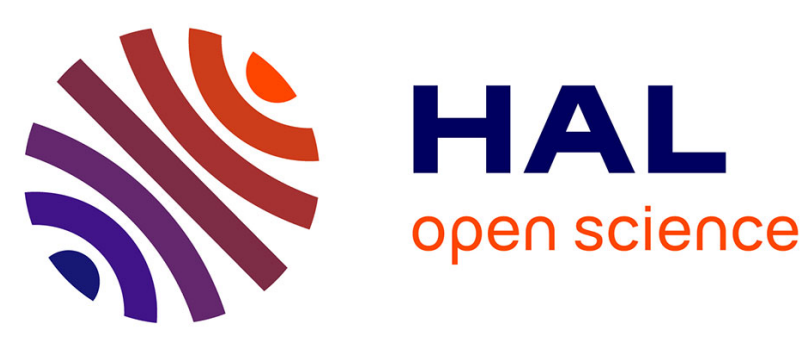

\title{
Etude de l'orientation cristallographique dans un silicium polycristallin massif
}

\author{
P. Andonov
}

\section{To cite this version:}

P. Andonov. Etude de l'orientation cristallographique dans un silicium polycristallin massif. Revue de Physique Appliquée, 1982, 17 (10), pp.657-679. 10.1051/rphysap:019820017010065700 . jpa00245044

\section{HAL Id: jpa-00245044 https://hal.science/jpa-00245044}

Submitted on 1 Jan 1982

HAL is a multi-disciplinary open access archive for the deposit and dissemination of scientific research documents, whether they are published or not. The documents may come from teaching and research institutions in France or abroad, or from public or private research centers.
L'archive ouverte pluridisciplinaire HAL, est destinée au dépôt et à la diffusion de documents scientifiques de niveau recherche, publiés ou non, émanant des établissements d'enseignement et de recherche français ou étrangers, des laboratoires publics ou privés. 
Classification

Physics Abstracts

$61.00-81.70-82.45$

\title{
Etude de l'orientation cristallographique dans un silicium polycristallin massif
}

\author{
P. Andonov \\ Laboratoire de Magnétisme, C.N.R.S., 92190 Bellevue, France \\ et Laboratoire de Marcoussis CR C.G.E.-DMT, Route de Nozay, 91460 Marcoussis, France
}

(Reçu le 18 mai 1982, révisé le 5 juillet 1982, accepté le 12 juillet 1982)

\begin{abstract}
Résumé. - L'étude de l'orientation cristallographique d'un silicium polycristallin obtenu par solidification directionnelle dans un creuset de graphite est reportée dans ce travail. Les techniques usuelles de diffraction des rayons $\mathrm{X}$ par réflexion ont tout d'abord été utilisées. Des variations de paramètres atteignant $14 \times 10^{-4} \AA$ ont été mises en évidence entre les différentes zones de cristallinite du lingot. La croissance des grains, parallèlement aux gradients de température les plus importants, produit dans le centre du lingot une structure axiale colonnaire avec des orientations privilégiées [211] et [111]. Sur les bords du creuset les orientations de croissance sont différentes. Les orientations [110] et [100] fortement défavorisées augmentent quand le taux d'impuretés $(\mathrm{Al}+\mathrm{B})$ devient supérieur à $9 \times 10^{15}$ atomes $/ \mathrm{cm}^{3}$.

Une méthode d'analyse basée sur l'attaque électrochimique du silicium a été ensuite développée. L'analyse du dépôt a été faite par diffraction des rayons $\mathrm{X}$ et par analyse ionique. Une corrélation entre son épaisseur et la densité réticulaire du plan de surface attaqué a été établie. La texture de solidification peut ainsi être représentée sans précision mais très rapidement par la carte des couleurs de lame mince observée après l'attaque électrolytique. Cette méthode semble bien adaptable pour un contrôle rapide d'une élaboration industrielle.
\end{abstract}

\begin{abstract}
An analysis of the crystallographic orientation of a polycrystalline silicon ingot obtained by directional solidification in carbon crucible is reported. To start with the usual methods of X-rays diffraction by reflection are used. Lattice constant deviations have been observed between the different crystalline zones of the ingot. The grain growth, in alignment with the higher temperature gradient, produces an axially columnar structure in the middle of the ingot, of mainly [211] and [111] orientations. The growth orientation is different at the crucible walls. When the impurities $(\mathrm{Al}+\mathrm{B})$ are more than $9 \times 10^{15}$ atoms. $\mathrm{cm}^{-3},[110]$ and [100] orientations are increased.

A electrochemical method with anodic dissolution of silicon has been developed. The deposit thus obtained was investigated by X-ray diffraction and S.I.M.S. A correlation between the film thickness and the reticular density of the etched surface was obtained. So the solidification texture can be shown, without a great precision but very rapidly, by a coloured card with interference colours of the thin anodic layer. This method suits well control of an industrial preparation.
\end{abstract}

1. Introduction. - La conversion directe de l'énergie solaire en énergie électrique à l'aide de photopiles au silicium fait actuellement l'objet d'intenses recherches tant pour définir les procédés de fabrication des cellules que pour élaborer un matériau de coût réduit [1].

Le silicium monocristallin de très haute pureté et de rendement photovoltaïque voisin de $18 \%$ pourrait être remplacé par un matériau polycristallin de pureté moindre à condition que les rendements de conversion restent satisfaisants $(\cong 10 \%)$. De nombreux procédés ont été développés, axés :

- soit sur l'élaboration directe des couches sensibles : dépôt en couche mince sur ruban; polycristal- lin autosupporté [2,3] ou sur substrat de carbone [4] ou de céramique [5], etc... Les techniques à mettre en œuvre sont délicates mais ne nécessitent qu'une très faible quantité de silicium,

- soit sur l'élaboration sous forme de lingots massifs dans lesquels seront découpées des plaquettes de faible épaisseur. Les techniques de tirage doivent rester simples et rapides pour que le coût de production soit faible comparé à celui des monocristaux élaborés par la méthode de Czochralsky et vue l'importante quantité de matériau nécessaire, il faut qu'un silicium de pureté moindre puisse être utilisé $[6,7]$.

C'est le but de l'étude entreprise par la Compagnie 
Générale d'Electricité aux Laboratoires de Recherche de Marcoussis [8]. Les caractéristiques électriques, chimiques et cristallographiques du matériau polycristallin ainsi obtenu, dont la composition et l'aspect cristallin varient dans le volume même du lingot, doivent être connues avant de procéder à la fabrication des cellules. Il a été montré sur un matériau comparable $[9,10]$ que le rendement photovoltaïque dépendait des « zones de cristallinités » du lingot. Il est donc intéressant de vérifier, s'il existe dans un tel matériau, des orientations de croissance cristalline privilégiées.

Ce travail décrit les méthodes mises en œuvre pour déterminer les propriétés cristallographiques du matériau. Après un exposé rapide des résultats obtenus à partir des techniques habituelles de diffraction des rayons $\mathrm{X}$, une méthode basée sur l'observation optique d'une couche mince obtenue par attaque électrolytique du silicium a été développée. Ce procédé de mise en œuvre aisée et non destructif, permet rapidement de connaître les orientations cristallines privilégiées à la surface des plaquettes et de mettre en évidence leurs défauts. Une sélection du matériau peut ainsi être assurée rapidement.

2. Conditions expérimentales. - 2.1 DESCRIPTION DU MATÉRIAU ÉTUDIÉ. - Le silicium fondu en creuset de graphite est refroidi rapidement dans un gradient thermique selon le procédé de Bridgman sans germe. Une solidification directionnelle est mise en évidence par la présence de nombreux grains à caractère basaltique orientés parallèlement au gradient de température le plus important, c'est-à-dire suivant l'axe longitudinal du four, leur longueur pouvant atteindre 2 à 3 centimètres suivant cet axe.

A la périphérie des blocs, on observe une croissance d'aspect dendritique d'orientation radiale. Plusieurs zones de "cristallinités différentes » apparaissent dans le lingot comme c'est le cas pour les matériaux "Silso " élaborés par Waker [6] et IBM [7] (Voir Fig. 1 et photos $1 a$ et $1 b$ ).

Pour la réalisation des cellules, des plaquettes minces (300 à $400 \mu \mathrm{m})$ sont sciées dans le lingot perpendiculairement à l'axe longitudinal. Après sciage,

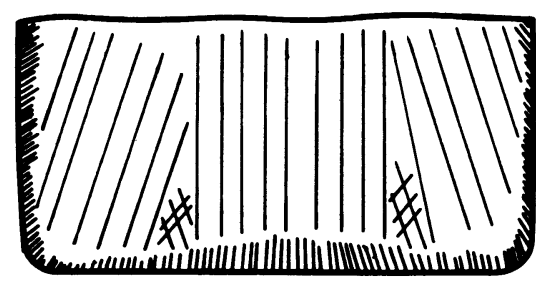

Fig. 1. - Section longitudinale d'un lingot C.G.E. Définition des zones de cristallinités différentes : ШWI Structure colonnaire, $\mathbb{U}$ Structure de transition, Structure intermédiaire,

[Longitudinal section of a polycrystalline silicon " C.G.E. » lingot : Ш Columnar structure, Шய Transition structure, Interjacent structure, שendritic structure.]

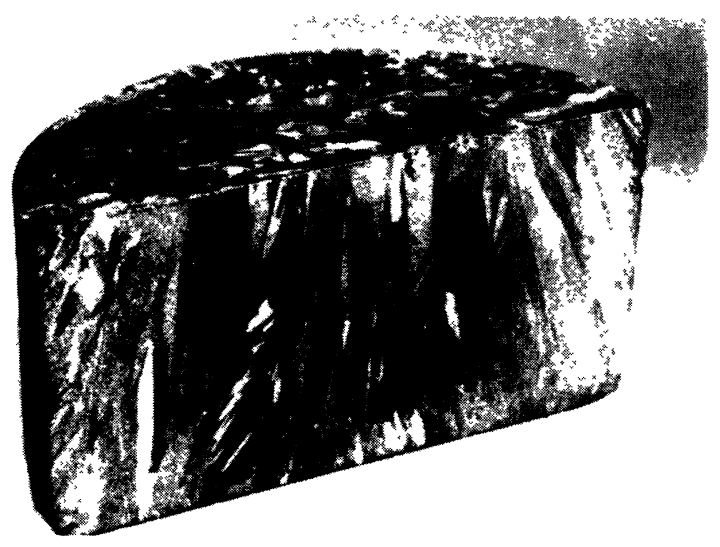

(a)

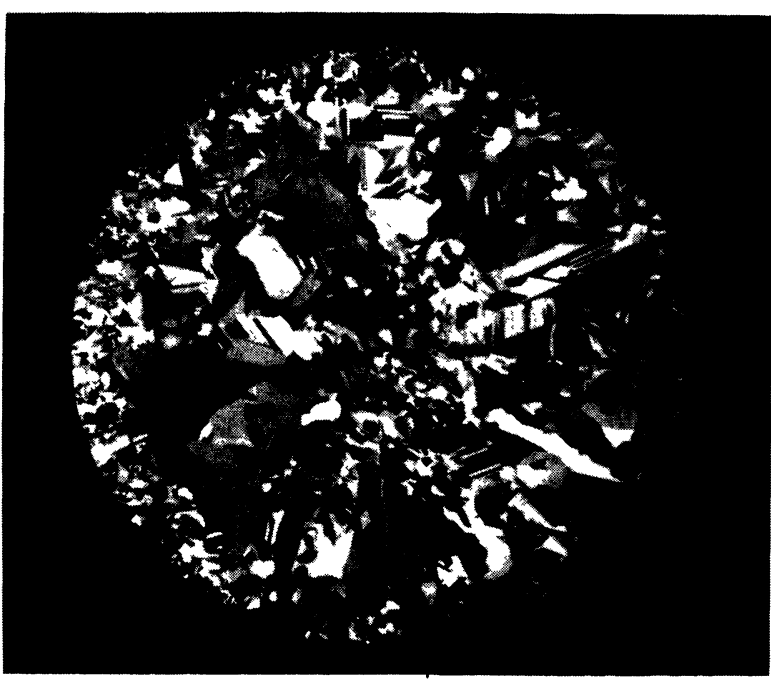

(b)

Photo 1. $-a$ ) Section longitudinale d'un lingot C.G.E b) Section transversale d'un lingot C.G.E.

[a) Longitudinal section of a "C.G.E. » ingot. b) Transversal section of a «C.G.E. » ingot.]

les surfaces sont polies (pâte diamantée $1 / 4 \mu \mathrm{m}$ ) puis attaquées dans des solutions diverses :

- pour révéler les grains :

$\left\{\begin{array}{l}\text { r } \\ \text { So }\end{array}\right.$

réactif de Secco :

$3 \mathrm{HNO}_{3}+2 \mathrm{CH}_{3} \mathrm{COOH}+2 \mathrm{HF}(2$ à $3 \mathrm{~min}$. $)$

Solutions $\mathrm{HF}+\mathrm{HNO}_{3}$ ou $\mathrm{HF}+\mathrm{CH}_{3} \mathrm{COOH}$

- pour révéler les macles et les dislocations :

( 1 vol. $\mathrm{H}_{2} \mathrm{Cr}_{2} \mathrm{O}_{7}\left(0,15\right.$ mol. dans $\left.\mathrm{H}_{2} \mathrm{O}\right)+$

+2 vol. HF (20 min.)

Solution CP4 :

$3 \mathrm{HF}+5 \mathrm{HNO}_{3}+3 \mathrm{CH}_{3} \mathrm{COOH}$ (2 à $3 \mathrm{~min}$.).

La surface des plaquettes est constituée de nombreux grains de taille variant de quelques $1 / 10 \mathrm{~mm}^{2}$ à plusieurs $\mathrm{mm}^{2}$ et présentant de nombreuses macles 


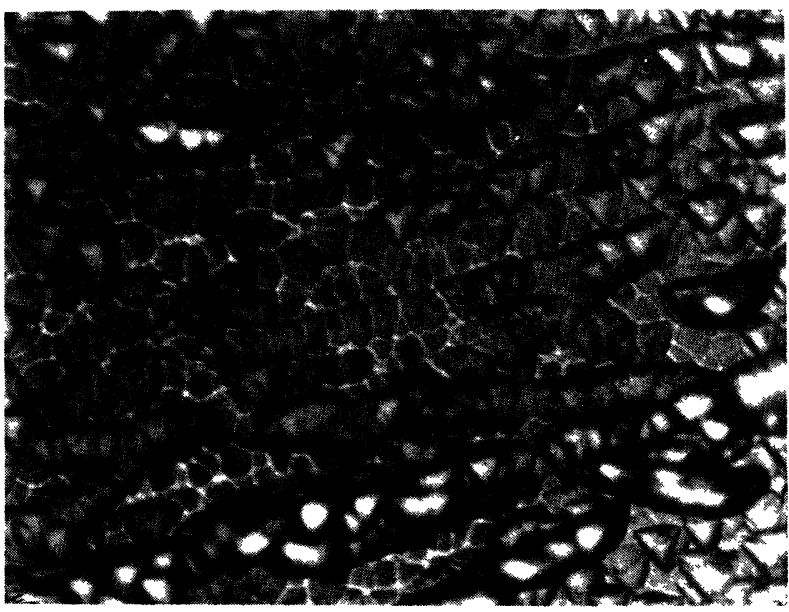

Photo 2. - Taux de dislocations mesuré au microscope optique $\mathrm{G} \times 1000=\left(10^{5} / \mathrm{cm}^{2}\right):($ surface attaquée $(111))$ et pollution $\mathrm{C}$ ou $\mathrm{SiC}$.

[Density of dislocations obtained with optical microscope $\mathrm{G} \times 1000=\left(10^{5} / \mathrm{cm}^{2}\right):$ etched surface $=$ plane $(111)$ and pollution $\mathrm{C}$ or $\mathrm{SiC}$.] et des taux de dislocations relativement importants $\left(10^{5}\right.$ à $\left.5 \times 10^{6} / \mathrm{cm}^{2}\right)$. Leur répartition en forme et en contraste est quelconque. Les joints de grains sont le plus souvent perpendiculaires à la surface.

Les méthodes d'attaque révèlent correctement les défauts (voir photo 2), mais l'observation au microscope des figures de corrosion ne permet pas, comme c'est le cas pour le silicium monocristallin, de définir les orientations cristallines; les contraintes importantes dans le matériau conduisant à des figures trop déformées sur la plupart des grains.

Ces études ont porté sur des échantillons prélevés dans différents lingots; leurs caractéristiques sont décrites dans le tableau I.

2.2 TeChNiQues DE DIFFRACTION UTILISÉES. - Les orientations cristallines à la surface des plaquettes ont été déterminées par diffraction des rayons $\mathrm{X}$ en utilisant des montages en réflexion.

L'épaisseur des échantillons reste toujours suffisante pour être considérée comme infinie quelle que soit la radiation utilisée. $\lambda \mathrm{CuK} \alpha$ est particulièrement

Tableau I. - Définition des échantillons étudiés

[Description of the observed samples.]

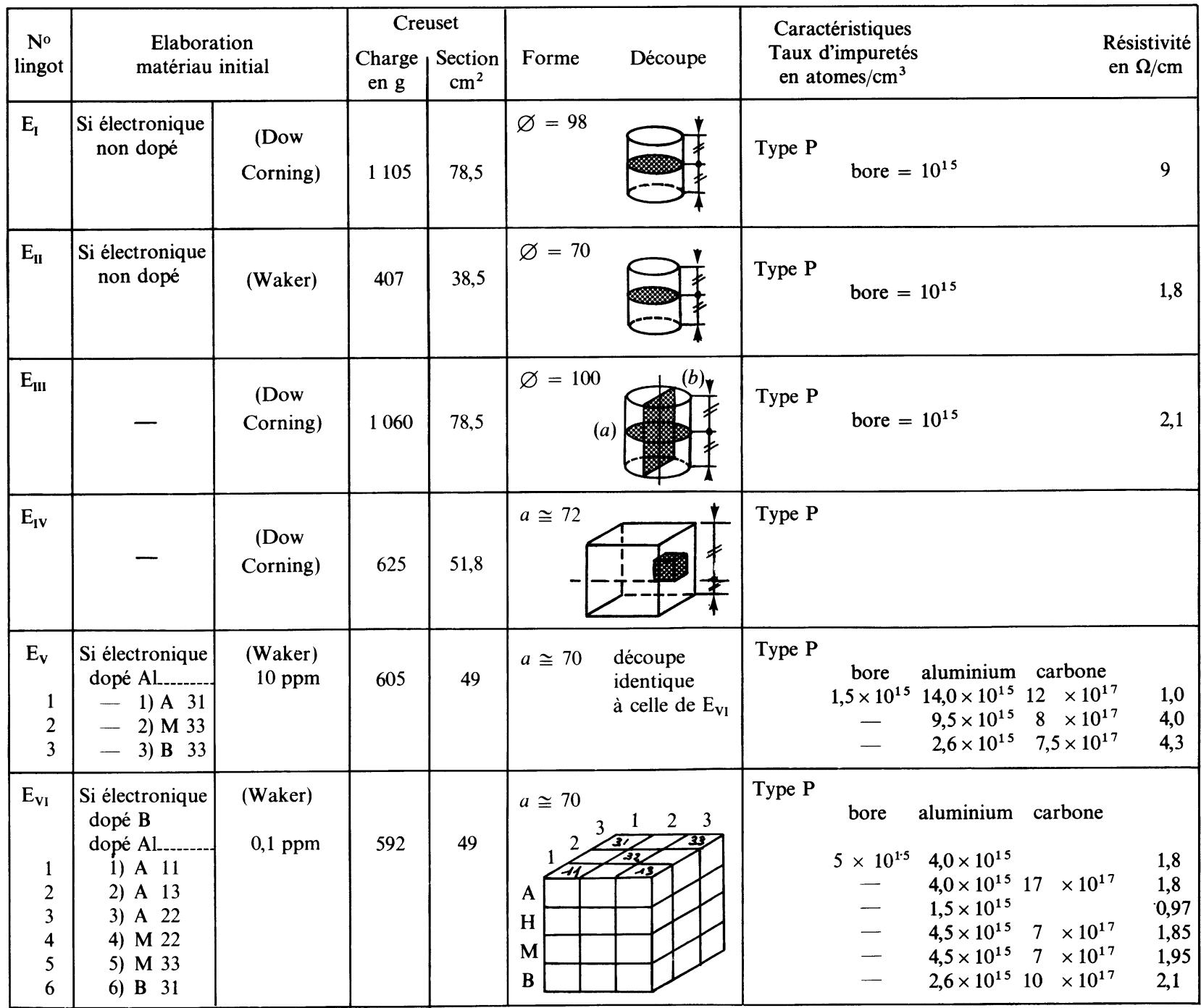


intéressante pour cette étude, puisque l'« épaisseur infinie » (absorption $\cong 99 \%$ de l'intensité incidente) égale $300 \mu \mathrm{m}$, soit l'épaisseur prévue pour les plaquettes (la même absorption est obtenue avec $160 \mu \mathrm{m}$ pour $\lambda \mathrm{CoK} \alpha$ et $300 \mu \mathrm{m}$ pour $\lambda \operatorname{MoK} \alpha$ ).

2.2.1 Enregistrement continu en $(\theta-2 \theta)$. - Sans possibilité de désorientation entre cristal et détecteur, ces mesures sont faites sur un équipement standard Philips : (générateur PW 1010, tube à anticathode de cuivre, foyer normal, goniomètre à axe horizontal et compteur proportionnel). Sept pics de Bragg peuvent être enregistrés correspondant aux réflexions successives $111,220,311,400,311,422$ et 511 ou 333 . Une réflexion n'est obtenue que si les plans d'indices convenables sont très faiblement désorientés par rapport à la surface. Même si cette condition est réalisée, la surface minimale explorée $\left[\cong 5 \times 2 \mathrm{~mm}^{2}\right.$ pour la réflexion 111] reste trop importante pour permettre une investigation précise de l'orientation en surface. L'enregistrement continu présente cependant un avantage important puisque l'apparition des pics parasites peut mettre en évidence la présence d'impuretés.

2.2.2 Distribution statistique des orientations en surface. - Cette étude a été faite sur un goniomètre Siemens à axe vertical avec le rayonnement monochromatique $\operatorname{CoK} \alpha_{1}$ (monochromateur à cristal de quartz interposé dans le faisceau incident). Détecteur et échantillon peuvent être désolidarisés. A l'aide d'un support intermédiaire, un échantillon de grandes dimensions $(1 / 2$ plaquette $\varnothing=100 \mathrm{~mm})$ peut être monté sur la tête goniométrique. Avec la géométrie utilisée, la surface irradiée dans le plan perpendiculaire au faisceau incident passant par l'axe du goniomètre est un rectangle de $20 \mathrm{~mm}$ de hauteur et de $0,5 \mathrm{~mm}$ de largeur, soit pour la réflexion $(h k l)$ une surface irradiée égale à $(20 \times 0,5) / \sin \theta_{h k l}$ en $\mathrm{mm}^{2}$. Géométrie et radiation limitent l'exploration à 6 orientations de plans réflecteurs (voir Tableau IV).

Pour chaque réflexion explorée, le détecteur est bloqué sur $2 \theta_{h k l}$ et l'échantillon désorienté autour de l'axe du goniomètre dans le domaine angulaire $\left[\theta_{h l k} \mp \Delta \theta_{\text {max }}\right]$ avec un pas d'exploration $\Delta \theta=0,20^{\circ}$ et une amplitude maximale $\Delta \theta_{\max }=20^{\circ}$. Pour chaque valeur $\theta$, l'échantillon est basculé autour de l'axe horizontal contenu dans la surface réfléchissante et passant par le centre de la tête goniométrique : basculement continu d'amplitude maximale $\Delta \alpha_{\max }= \pm 25^{\circ}$.

Lorsqu'une réflexion est obtenue la zone active de la surface réfléchissante est localisée en hauteur en déplaçant un diaphragme qui limite le faisceau incident. La translation verticale d'une fente étroite devant le détecteur permet de repérer sa surface utile et de déterminer l'azimuth du faisceau diffracté. L'orientation du plan réflecteur est alors déterminée avec une précision égale à $\pm 0,2^{\circ}$ en $\theta$ et $\pm 0,3^{\circ}$ en $\alpha$.

Les ordonnées et dimensions verticales des grains entrant en réflexion sont évaluées à $\pm 0,3 \mathrm{~mm}$; les abscisses et dimensions latérales avec la même incer- titude sauf pour les réflexions 111 dans le domaine angulaire $\left|\theta_{111} \pm 5^{\circ}\right|$.

2.2.3 Diagramme de Laue en retour.-L'orientation cristalline est déterminée à l'aide de diagrammes de Laue en retour. Le faisceau de rayons $X$, généré sous une puissance de $800 \mathrm{~W}(20 \mathrm{~mA} \times 40 \mathrm{kV}$, fourni par un générateur Philips 1008) est collimaté afin que la surface irradiée sur l'échantillon reste inférieure à $0,5 \mathrm{~mm}^{2}$. Les taches sont enregistrées sur un film plan situé à une distance égale à $30 \mathrm{~mm}$ de la surface réfléchissante.

Les hyperboles, reliant les taches les plus apparentes sur un cliché, correspondent à des plans d'indices simples. Le dépouillement est facilité par l'utilisation des abaques de Grenninger [11]. Dans les cas où le point d'impact du faisceau ne recouvre qu'un seul cristal non maclé et dont un axe important est peu désorienté par rapport au faisceau incident, l'identification des taches du diagramme est rapide (cliché Fig. 2).

Mais généralement, les orientations ne sont définies qu'à partir des projections stéréographiques en indexant un grand nombre de tâches du stéréogramme ([12], p. 318 et [13], p. 71 et 111). Souvent, sur un même cliché, il y a superposition de plusieurs diagrammes (cliché Fig. 3). Ceci provient de la difficulté d'isoler des grains de taille suffisante et de contours bien révélés à laquelle s'ajoute la présence de nombreux défauts (macles, joints de grains), qui peuvent participer à la diffraction. La séparation des tâches ne peut se faire qu'à partir de leurs formes; or l'élaboration en cristallisation rapide conduit à des contraintes importantes dans le matériau avec déformation des plans réflecteurs; les taches sont souvent floues. Ces remarques expliquent la difficulté du dépouillement des diagrammes. Enfin, si les cristallites sont orientés de telle façon qu'aucun axe de faibles indices ne soit voisin de celui du faisceau incident, les diagrammes de Laue en retour sont inexploitables.

L'orientation d'un grain à la surface de la plaquette est toujours définie par un plan de faibles indices (voir Tableau II) en notant les désorientations de la normale de celui-ci $(\tau, \sigma$ et $\rho$ ) par rapport au plan de surface perpendiculaire au faisceau incident (Fig. 4). La rotation du plan sur lui-même est définie par l'angle $\chi=\overline{N S, Y Y^{\prime}}$ que fait l'axe $N S$ de la projection stéréographique avec l'axe vertical $Y Y^{\prime}$ de la chambre.

Remarques. - Le pôle [111] est un des plus faciles à repérer sur les stéréogrammes. Sa position par rapport au centre de la projection stéréographique est reportée dans le tableau II. L'observation des deux dernières colonnes indique que la distinction entre plans (310) et (311) sera difficile à mettre en évidence à partir des différences de densité réticulaire, mais son ambiguité à partir du stéréogramme. Par contre, la distinction entre (231) et (331) restera incertaine puisque les différences entre les densités réticulaires et les projec- 
(111)

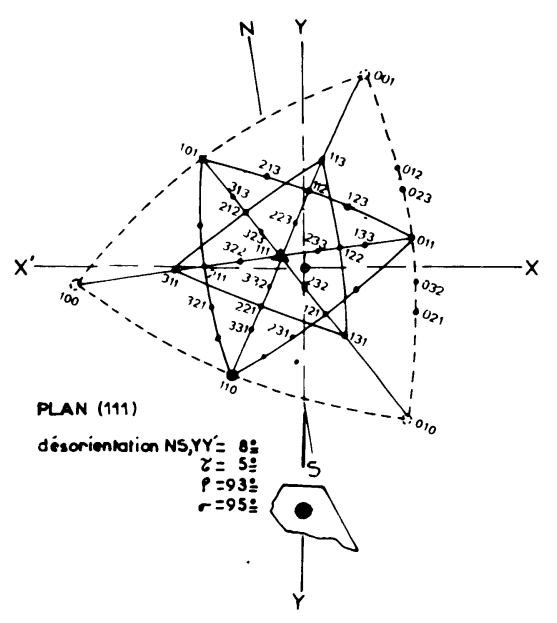

(311)
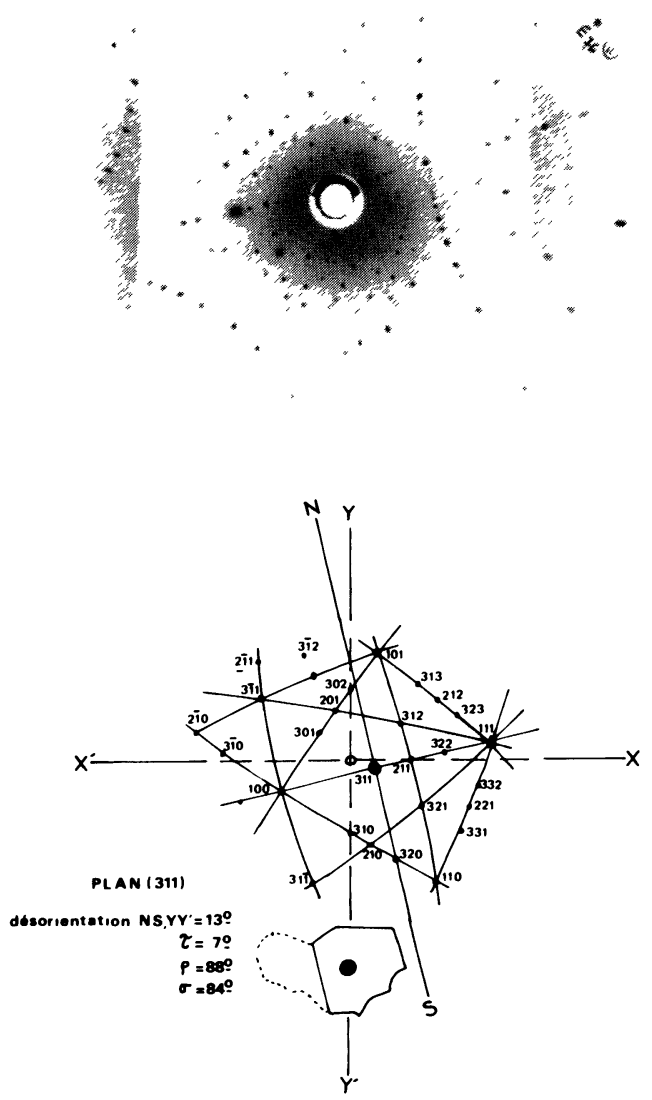

(211)

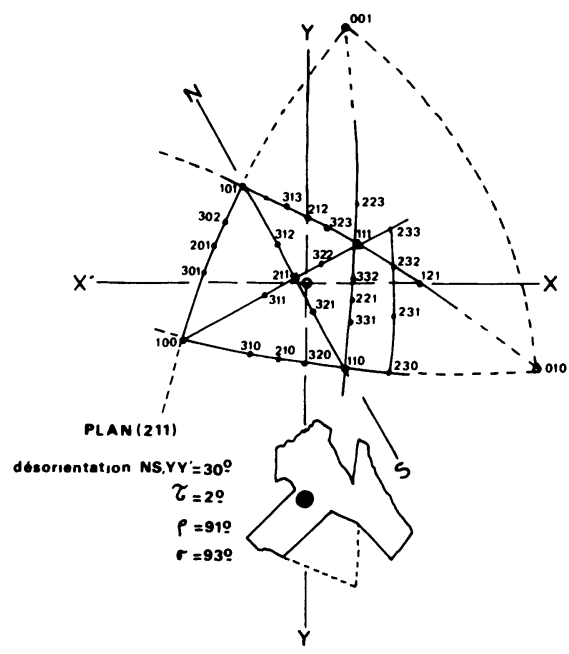

(331)
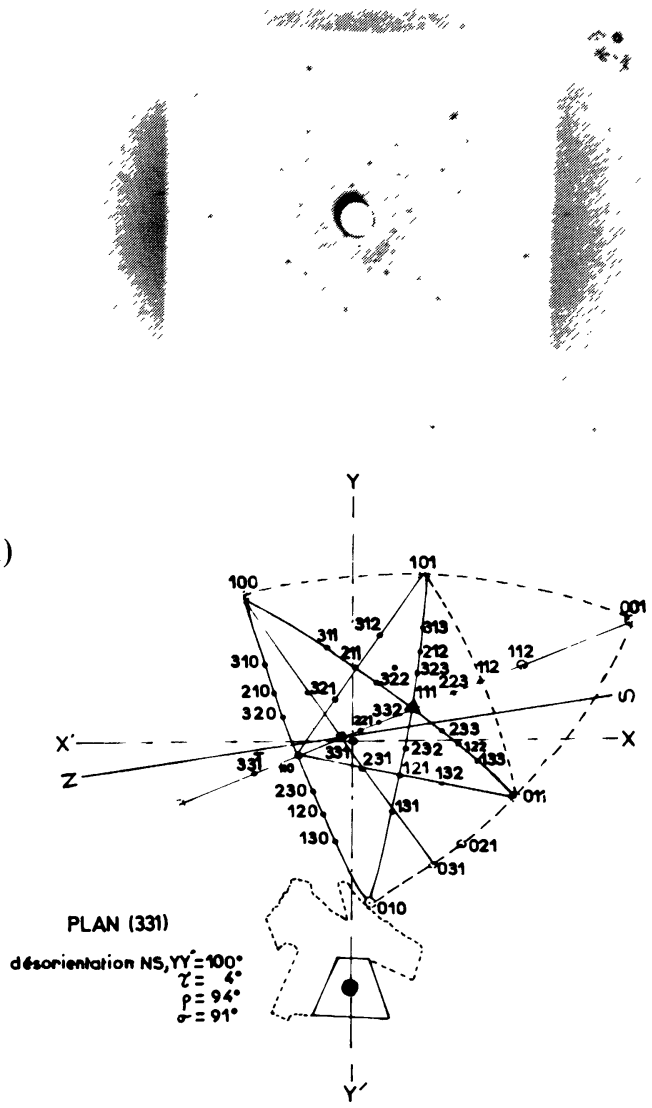

Fig. 2. - Cliché Laue et projection stéréographique (111), (211), (311), (331).

[Back-reflection Laue photographs and stereographic projections (111), (211), (311), (331).] 


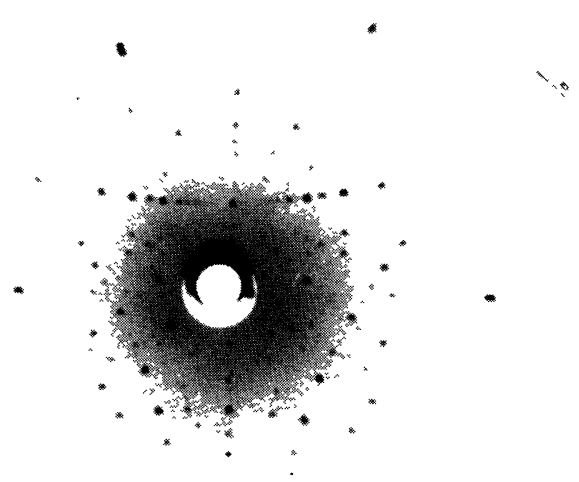

a)
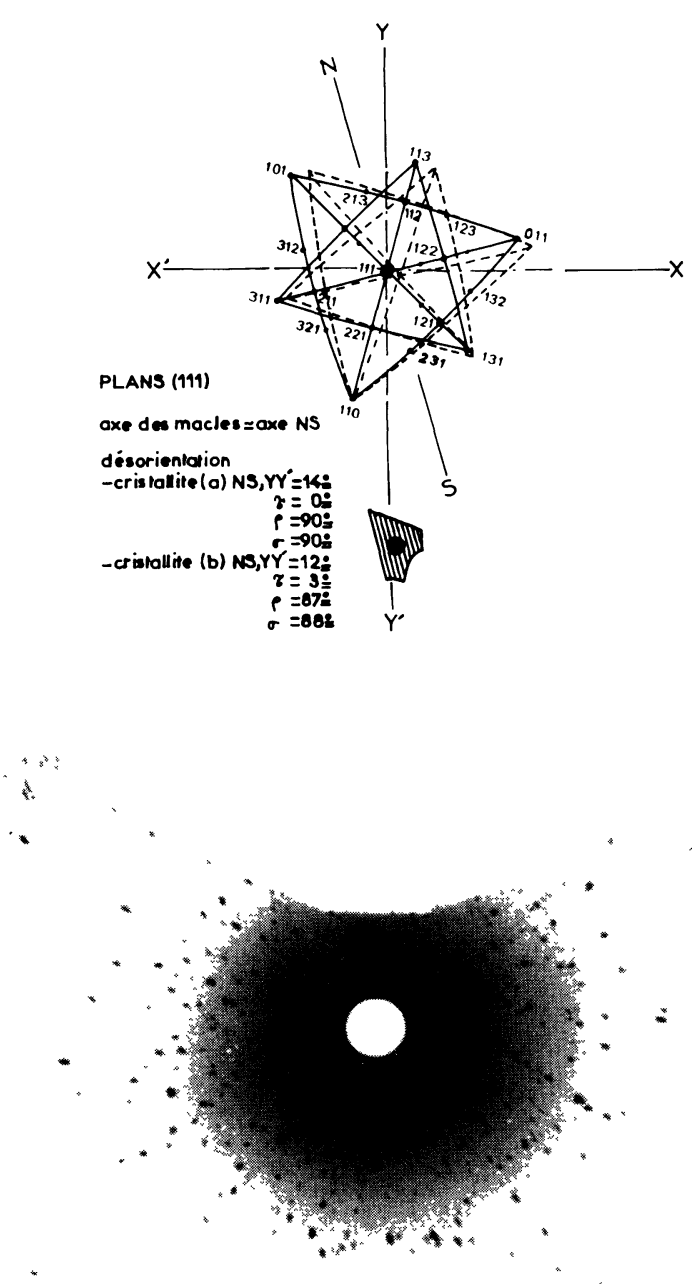

b)

Fig. 3. - Cliché Laue : a) superposition de plusieurs diagrammes (111) macle; $b$ ) cliché inextricable.

[Back-reflection Laue photographs : a) superposition of two diagrams ; twinned crystal : plane $(111) ; b$ ) inextricable diagram; planes : (211), (311), with many no indiced spots.]

tions stéréographiques sont faibles. Enfin, les plans (331) et (210) possèdent la même densité réticulaire.
- Superposition de plusieurs diagrammes sur un même cliché :

* La zone irradiée recouvre deux ou trois cristallites. Les cas les plus fréquents observés sont :

$$
\begin{aligned}
& \text { plan }(111)+\text { plan }(211) \\
& \text { plan }(111)+\text { plan }(311) \\
& \text { plan }(111)+2 \text { plans }(211) \text { désorientés entre eux } \\
& \text { plan }(311) \text { + plan (110) }
\end{aligned}
$$

En général, les plans repérés présentent, par rapport à la surface, des désorientations qui s'expliquent en tenant compte des angles interplanaires du système cubique. Aucune condition restrictive de maclage n'existe dans ce système, mais le plus souvent les plans de macle sont les plans (111).

Dans le dernier exemple, les angles interplanaires $(110) /(311),(110) /(110)$, ou $(311) /(311)$ expliquent bien les orientations observées sur le stéréogramme, mais les taches présentent un astérisme très marqué, particulièrement celles correspondant au plan (110), ce qui peut s'expliquer par une déformation importante des plans réticulaires. Cette association correspond à des domaines pour lesquels les réponses en E.B.I.C. et en photocourant restent faibles.

** La zone irradiée recouvre un cristallite maclé. Exemple : grain no 7 (Fig. 19) qui présente des stries noires parallèles et serrées : traces des plans de macle sur la surface. Les deux diagrammes correspondent à deux plans (111) très faiblement désorientés l'un par rapport à l'autre $\left(\chi<5^{\circ}\right)$ (voir Fig. 3), sans que n'apparaissent d'autres orientations. Les angles interplanaires $(111) /(111)$ ne peuvent expliquer les résultats obtenus; les macles d'association mal définies ou le volume des joints n'apparaissent pas sur le cliché. Les taches sont à la fois discontinues et déformées. La présence de cristaux polygonisés, c'est-à-dire formés d'agrégats de petits blocs très légèrement désorientés entre eux et des plans réticulaires distordus peuvent conduire à ces particularités.

Avec la croissance colonnaire observée dans les lingots, les plans de mâcle déformés pourraient stabiliser la cristallisation verticale en éliminant en partie les tensions internes créées dans le matériau au cours du refroidissement rapide.

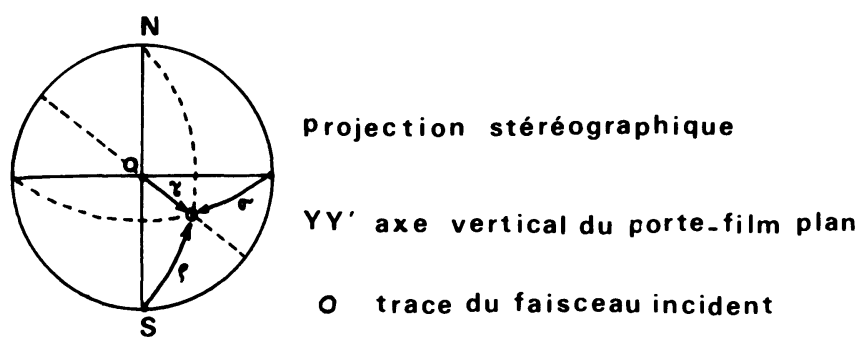

Fig. 4. - Projection stéréographique : définition des désorientations. Projection stéréographique. $Y Y^{\prime}$ axe vertical du porte-film plan, $O$ trace du faisceau incident.

[Stereographic projection : definition of the disorientations.] 
Tableau II. - Caractéristiques des plans réflecteurs du cubique diamant

[Characteristics of the reticular planes in the cubic diamond.]

\begin{tabular}{|c|c|c|c|c|c|c|c|}
\hline \multirow[t]{2}{*}{$\begin{array}{l}\text { Orienta- } \\
\text { tion du } \\
\text { plan }\end{array}$} & \multirow[t]{2}{*}{$\begin{array}{l}\text { Indices } h k l \\
\text { des } \\
\text { réflexions }\end{array}$} & \multirow[t]{2}{*}{$\begin{array}{l}\text { Distance } \\
\text { réticulaire } \\
\text { en } \AA\end{array}$} & \multirow[t]{2}{*}{$\begin{array}{l}\text { Nombre de plans } \\
\text { d'espacement } \\
\text { équivalent }\end{array}$} & \multirow[t]{2}{*}{$\left|\frac{F_{n k l}}{f}\right|^{2}$} & \multirow[t]{2}{*}{$\begin{array}{l}\text { Densité réti- } \\
\text { culaire } \\
\text { calculée en } \\
\text { atome } / \AA^{2}\end{array}$} & \multicolumn{2}{|c|}{$\begin{array}{c}\text { Position du pôle (111) } \\
\text { par rapport au centre } 0+ \\
\text { de la projection stéréo- } \\
\text { graphique }\end{array}$} \\
\hline & & & & & & $r$ en $\mathrm{mm}$ & $\alpha$ en ${ }^{o}$ \\
\hline 110 & 220 & 1,92038 & 12 & 64 & 0,0959 & 33 & 0 \\
\hline 111 & 111 & 3,13528 & 8 & 34 & 0,0783 & 0 & 0 \\
\hline 100 & 400 & 1,35773 & 6 & 64 & 0,0678 & 55 & 45 \\
\hline 211 & 422 & 1,10862 & 24 & 64 & 0,0554 & & 90 \\
\hline 310 & 620 & 0,85869 & 24 & 64 & 0,0429 & 42 & -33 \\
\hline 311 & 311 & 1,63734 & 24 & 32 & 0,0407 & 27 & 90 \\
\hline 231 & 462 & 0,72574 & 48 & 64 & 0,0362 & 21 & 48 \\
\hline 331 & 331 & 1,24597 & 24 & 32 & 0,0303 & 21 & 13 \\
\hline 210 & 840 & 0,60719 & 24 & 32 & 0,0303 & 37,5 & -25 \\
\hline 221 & 884 & 0,45258 & 24 & 32 & 0,0220 & 14 & 18 \\
\hline 320 & 1280 & 0,37657 & 24 & 32 & 0,0188 & 21 & 16 \\
\hline 322 & 1288 & 0,32929 & 24 & 32 & 0,0164 & 10 & 90 \\
\hline
\end{tabular}

Le silicium possède une structure cubique diamant, seules apparaissent les réflexions pour lesquelles les indices $h, k, l$, sont de même parité et tels que $h+k+l=2 n+1$ ou $h+k+l=4 n$.

Les caractéristiques sont reportées par valeur décroissante de la densité réticulaire. Distance et densité ont été calculées pour un paramètre $a=5,430941 \AA$ [14] et une densité atomique $N=0,049942$ atome $/ \AA^{3}$ ( 8 atomes par maille).

L'orientation d'un plan, à partir des diagrammes de Laue, est définie avec une imprécision $\leqslant \pm 2^{\circ}$.

2.2.4 Diffraction neutronique. - A la périphérie des blocs, dans la zone de cristallinité à structure dendritique (Fig. 1) la section des grains, à la surface des plaquettes, est trop faible pour que les méthodes ponctuelles précédentes soient utilisées. Cependant le volume de ces grains reste encore trop important pour qu'une répartition statistique non erronée soit décrite à partir d'analyses de texture par diffraction des rayons $\mathrm{X}$ (contrôle fait sur le goniomètre de texture Siemens avec un échantillon de surface $=25 \times 15 \mathrm{~mm}^{2}$, d'épaisseur $e=110 \mu \mathrm{m}$ : valeur optimale en transmission avec $\lambda \mathrm{CuK} \alpha$ ).

Par contre l'absorption des neutrons par le silicium est négligeable. Il est possible de travailler en transmission sur un échantillon de volume important (de l'ordre $\mathrm{du} \mathrm{cm}^{3}$ ). Une première expérience a été entreprise à l'Institut Laue-Langevin $\left({ }^{1}\right)$, sur le diffracto-

( $\left.{ }^{1}\right)$ En collaboration avec M. Pannetier. mètre D1B équipé d'un multidétecteur. L'exploration a été faite dans le plan équatorial, dans un domaine angulaire couvrant $80^{\circ}$ en $2 \theta$ (voir le schéma 5). Avec $\lambda=1,27 \AA, 9$ réflexions de Bragg sont enregistrées simultanément (de 6 à $86^{\circ}$ ); pour une orientation donnée de l'échantillon. Les spectres sont enregistrés pour 12 orientations différentes (rotation de l'échantillon sur lui-même autour de l'axe du spectromètre avec un pas $\Delta \theta=15^{\circ}$ ). Pour chaque orientation de l'échantillon, les plans $(h k l)$ entrant en réflexion sont repérés et les désorientations entre ces plans déterminées.

Ce montage simplifié, sans berceau d'Euler, ne permet pas l'étude de la texture proprement dite; mais la probabilité de présence des plans réflecteurs peut être calculée en mesurant l'aire sous chaque pic et en la normalisant par rapport à l'intensité théorique du pic équivalent obtenu avec un échantillon de poudre.

- Dans le cas de la diffraction des rayons X, avec un montage équivalent, les facteurs de normalisation sont calculés à partir de la formule :

$$
I_{h k l}=c n L p F^{2} D
$$




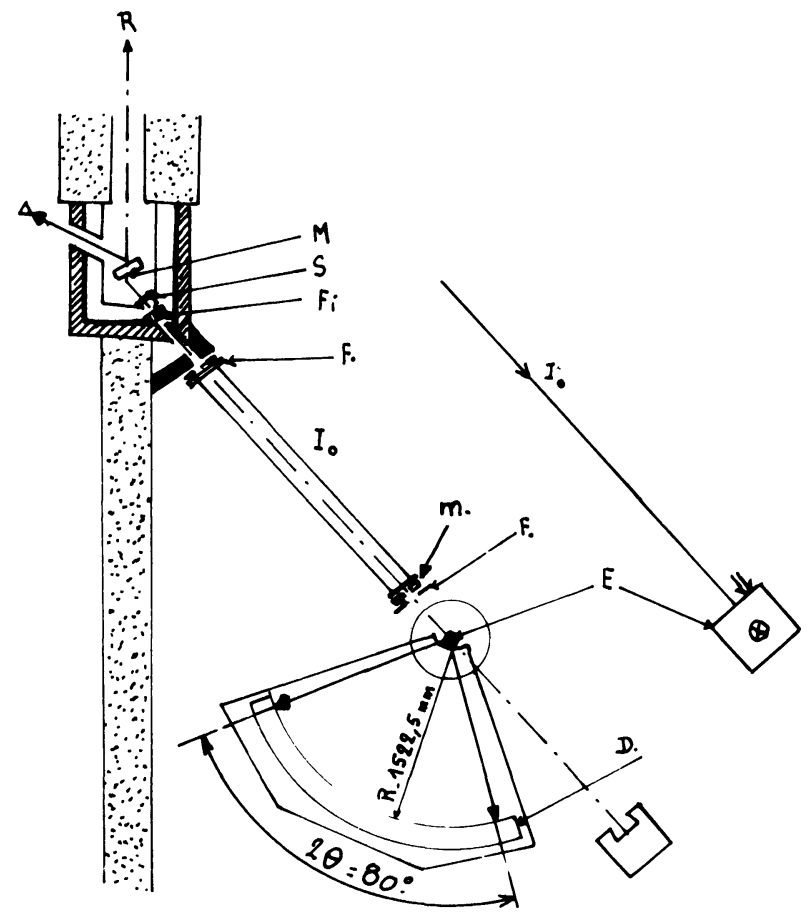

Fig. 5. - Schéma de principe du diffractomètre 2 axes D1B équipé d'un multidétecteur. Conditions expérimentales : $\lambda=1,27 \AA$ : Faisceau de neutrons $h=20 \mathrm{~mm}, l=16 \mathrm{~mm}$; temps de comptage : $15 \mathrm{~min}$./diagramme; distance échantillon-détecteur $=1,5225 \mathrm{~m}$; hauteur utile du détecteur $=$ $10 \mathrm{~cm}$; multicompteur : 400 cellules pour $80^{\circ}$ en $2 \theta$; $\mathrm{R}=$ réacteur $; \mathrm{M}=$ monochromateur graphite $; \mathrm{S}=$ obturateur; $\mathrm{Fi}=$ filtres de graphite; $\mathrm{F}=$ fenêtre ajustable; $\mathrm{m}=$ moniteur $; \quad \mathrm{E}=$ échantillon $; \mathrm{D}=$ multidétecteur ; $I_{0}=$ direction du faisceau incident $; \otimes:$ axe de l'échantillon parallèle à l'axe du lingot; $\checkmark l$ : face latérale du lingot.

[Two-axis diffractometer with multidetector D1B : Wavelength $=1.27 \AA$; neutron beam $h=20 \mathrm{~mm}, l=16 \mathrm{~mm}$; counting time : $15 \mathrm{~min} . /$ diagram; distance sample-detector $=1.5225 \mathrm{~m}$; working height of the detector $=10 \mathrm{~cm}$; multidetector : 400 units to $2 \theta=80^{\circ} ; \mathrm{R}=$ reactor; $\mathrm{M}=$ monochromator-graphite; $\mathrm{S}=$ beam shutter; $\mathrm{Fi}=$ graphite filters; $\mathrm{F}=$ adjustable window $; \mathrm{m}=$ monitor ; $\mathrm{E}=$ sample $; \mathrm{D}=$ multidetector $; \otimes:$ sample axis parallel to ingot axis ; $\downarrow \downarrow$ : lateral face of the ingot.]

où

$n$ : la multiplicité du plan $(h k l)$,

$L p$ : le facteur de Lorentz et polarisation,

$F$ : le facteur de structure,

$D$ : le facteur de Debye-Waller,

$c$ : une constante choisie pour que $I_{111}=100$ (raie la plus intense pour l'échantillon de poudre).

$(F$ est calculé à partir du facteur de diffusion atomique $f$ reporté dans les Tables de Cristallographie, vol. III, tableau 3.3.1A.)

- Dans le cas de la diffraction des neutrons, la normalisation précédente est simplifiée puisque seul intervient le facteur de Lorentz $L$ et que le facteur de structure $F$ est calculé à partir de la longueur de diffusion cohérente qui reste constante quel que soit l'angle de diffraction ( $b=4,15$ Fermi).

Les valeurs numériques de ces différentes grandeurs sont reportées dans le tableau $\mathrm{V}$.

3. Résultats. - 3.1 Mesures deS PARAMÈTres. Elles ont été faites sur l'équipement standard Philips. La méthode de Bradley et Jay ([12], p. 223) a permis de déterminer les paramètres des différents échantillons de silicium avec une précision de $\pm 3 \times 10^{-4} \AA$.

Les mesures ont porté sur :

- des monocristaux étirés à partir de Si de qualité électronique non dopé (plans de surface (111) et (400)),

- des plaquettes taillées dans le lingot $\mathrm{E}_{\mathrm{II}}$. Des surfaces réfléchissantes ont été isolées sur des grains de taille suffisante pour être considérés comme monocristaux. Aucune mesure ne peut être faite sur les bords du lingot; domaine de croissance en fines dendrites,

- des échantillons de poudre, obtenus en broyant et tamisant $(\varnothing<50 \mu \mathrm{m})$ des blocs de $0,15 \mathrm{~cm}^{3}$ prélevés sur le lingot $E_{V I}$ en $A_{11}, A_{22}$ et $M_{22}$,

- un échantillon de poudre standard fourni par Philips pour l'étalonnage du goniomètre.

Le réglage est contrôlé et affiné. Ainsi est obtenue avec $\lambda \mathrm{CuK} \alpha_{1}$ et $\lambda \mathrm{CuK} \alpha_{2}$, la valeur du paramètre récemment déterminé sur des monocristaux non dopés $a=5,430941 \AA, \Delta a / a=2 \times 10^{-6}$ [14].

Les résultats sont reportés dans le tableau III.

Pour les prélèvements faits dans le centre des lingots, zone de cristallinité colonnaire, les paramètres mesurés restent constants (écarts $\cong 2$ à $3 \times 10^{-4} \AA$ compris dans les limites de précision). Par contre, dans les zones de cristallinités différentes, les écarts de paramètre sont plus importants

$$
\left\{\begin{array}{l}
\mathrm{d} a \cong-7 \times 10^{-4} \AA \text { dans la zone de structure de } \\
\text { transition, } \\
\mathrm{d} a \cong-14 \times 10^{-4} \AA \text { dans la zone de structure den- } \\
\text { dritique. }
\end{array}\right.
$$

Ces écarts ne peuvent s'expliquer par des différences de taux d'impuretés. Malgré la ségrégation de l'Al ou autres impuretés, au sommet du lingot, les taux d'impuretés restent trop faibles pour faire varier le paramètre. La diminution du paramètre est due aux contraintes résiduelles importantes principalement sur le bord des lingots où les vitesses de refroidissement sont élevées.

3.2 EnREGistrement CONTINU $(\theta-2 \theta)$. - Les résultats obtenus sont peu nombreux et restent qualitatifs. Un enregistrement rapide obtenu avec $\mathrm{E}_{\mathrm{II}}$ ne présente que les réflexions [111] et [333] mais étroites et très intenses. Une détermination très approximative indique que $60 \%$ de la surface est occupée par des plans (111) peu désorientés : résultat confirmé par des diagrammes de Laue. 
Tableau III. - Variations des paramètres dans le lingot

[Lattice constant variations in the P. silicon ingot « C.G.E. ».]

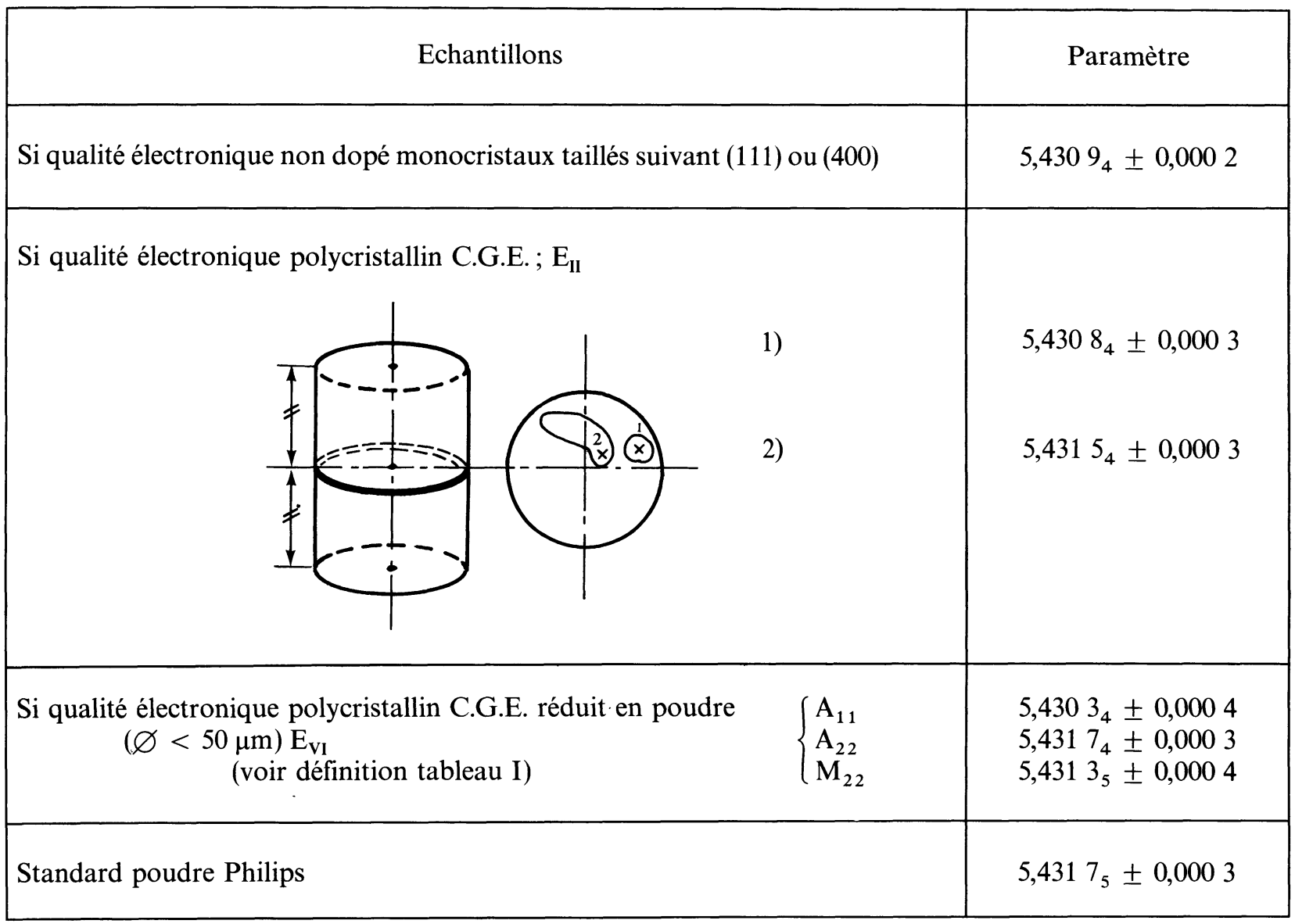

Avec l'échantillon $\mathrm{E}_{\mathrm{l}}$, les enregistrements ne présentent aucun pic de Bragg à l'exception d'une réflexion large et de très faible intensité autour de $2 \theta_{422}$. Des diagrammes de Laue et l'étude de la distribution statistique des orientations en surface, confirment l'existence de plans réflecteurs (211) mais désorientés de quelques degrés par rapport à la surface (voir 3.3).

Cette méthode n'est qu'une première observation rapide des plaquettes. Une surface maximum de l'échantillon est irradiée afin d'enregistrer le plus de réflexions possibles. Ainsi pour certains échantillons, une pollution de carbone a été décelée sous forme de graphite hexagonal (pollution due au creuset) et sous forme de carbure de silicium ( $\alpha \mathrm{SiC}$-hexagonal). Voir photo 2, agrégats de 3 à $5 \mu \mathrm{m}$, repérés en plusieurs régions des lingots.

\subsection{Distribution STATISTIQUE DES ORIENTATIONS} DE SURFACE. - L'étude a porté sur la plaquette $\mathrm{E}_{\mathrm{l}}$. Après attaque chimique, les grains présentent un contraste très marqué. Certains apparaissent d'un noir très brillant, avec un taux de dislocations compris entre $8 \times 10^{4}$ et $10^{5}$ par $\mathrm{cm}^{2}$, pour les autres, le taux est plus élevé : $4 \times 10^{5}$ à $10^{6}$ par $\mathrm{cm}^{2}$. A l'exception d'une

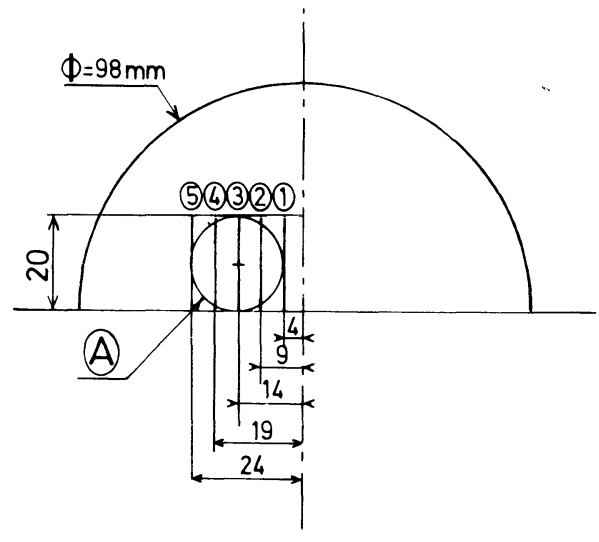

Fig. 6. - Plages explorées sur l'échantillon $\mathrm{E}_{\mathrm{II}} \cdot 1,2,3,4,5$ : distribution statistique; (A) : attaque électrochimique.

[Explored zones in the sample $\mathrm{E}_{\mathrm{II}}$. 1, 2, 3, 4, 5 : statistical distribution; A : electrochemical etching.]

dizaine de grains de surface comprise entre 5 et $10 \mathrm{~mm}^{2}$,

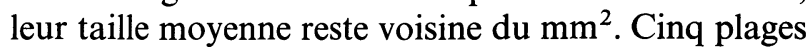
ont été explorées dont les positions sont indiquées figure 6. Les orientations en surface ont été déterminées pour 270 cristaux et sont reportées dans le tableau IV ainsi que la répartition en nombre. 
Tableau IV. - Distribution statistique des orientations de surface

[Statistical distribution of the surface orientations.]

\begin{tabular}{|c|c|c|c|c|c|c|c|c|}
\hline \multirow{2}{*}{$\begin{array}{l}\text { Indices } h k l \\
\text { des plans } \\
\text { réflecteurs }\end{array}$} & \multirow{2}{*}{$\begin{array}{c}\text { Surface irradiée } \\
\text { pour l'orientation } \\
\theta_{h k l}{\text { en } \mathrm{cm}^{2}}^{2}\end{array}$} & \multicolumn{5}{|c|}{$\begin{array}{l}\text { Nombre de cristaux } \\
\text { repérés dans les zones }\end{array}$} & \multirow{2}{*}{$\begin{array}{l}\text { Nombre total } \\
\text { de cristaux }\end{array}$} & \multirow{2}{*}{$\begin{array}{l}\text { Nombre moyen } \\
\text { de cristaux } \\
\text { réflecteurs }(h k l) \\
\text { en surface } \\
\text { pour } 1 \mathrm{~cm}^{2}\end{array}$} \\
\hline & & 1 & 2 & 3 & 4 & 5 & & \\
\hline 111 & 1,76 & 18 & 10 & 11 & 22 & 19 & 80 & 45,5 \\
\hline 220 & 1,07 & 0 & 2 & 14 & 2 & 3 & 21 & 19,6 \\
\hline 311 & 0,92 & 8 & 5 & 15 & 5 & 12 & 45 & 48,9 \\
\hline 400 & 0,76 & 6 & 2 & 2 & 2 & 3 & 15 & 19,7 \\
\hline 331 & 0,69 & 6 & 6 & 7 & 6 & 5 & 30 & 43,5 \\
\hline 422 & 0,62 & 13 & 6 & 12 & 27 & 21 & 79 & 127,4 \\
\hline
\end{tabular}

Les cristaux dont les plans de surface sont voisins de l'orientation (211) sont majoritaires. Ce sont les cristaux noirs brillants (contrôle par diagramme de Laue). Les plans (111), (311) et (331) se retrouvent avec la même probabilité de présence alors que les orientations (100) et (110) sont très défavorisées. Ni l'erreur statistique élevée (10 à $20 \%$ selon les orientations), ni la différence de multiplicité des plans d'espacement équivalent ne suffisent à expliquer ces écarts.

La même étude, faite sur la plaquette $\mathrm{E}_{\mathrm{III}(\mathrm{a})}$ a conduit à des résultats équivalentš, bien que l'aspect de la surface soit différent : grains de taille moyenne plus homogène, voisine de $1 \mathrm{~mm}^{2}$ et moins contrastés.

Quelle que soit la plaquette observée, sur la majorité de la surface (exception faite de la zone de cristallinité dendritique), les contours des grains ont des formes quelconques réparties au hasard. L'étude d'une plaque taillée parallèlement à l'axe du lingot $\left(\mathrm{E}_{\mathrm{III}(\mathrm{b})}\right)$ ne met pas en évidence de réflexions nettes. Ceci signifie qu'il n'y a pas d'orientation privilégiée de plans réflecteurs parallèles à l'axe du lingot. Cette observation est confirmée par les résultats des diagrammes de Laue

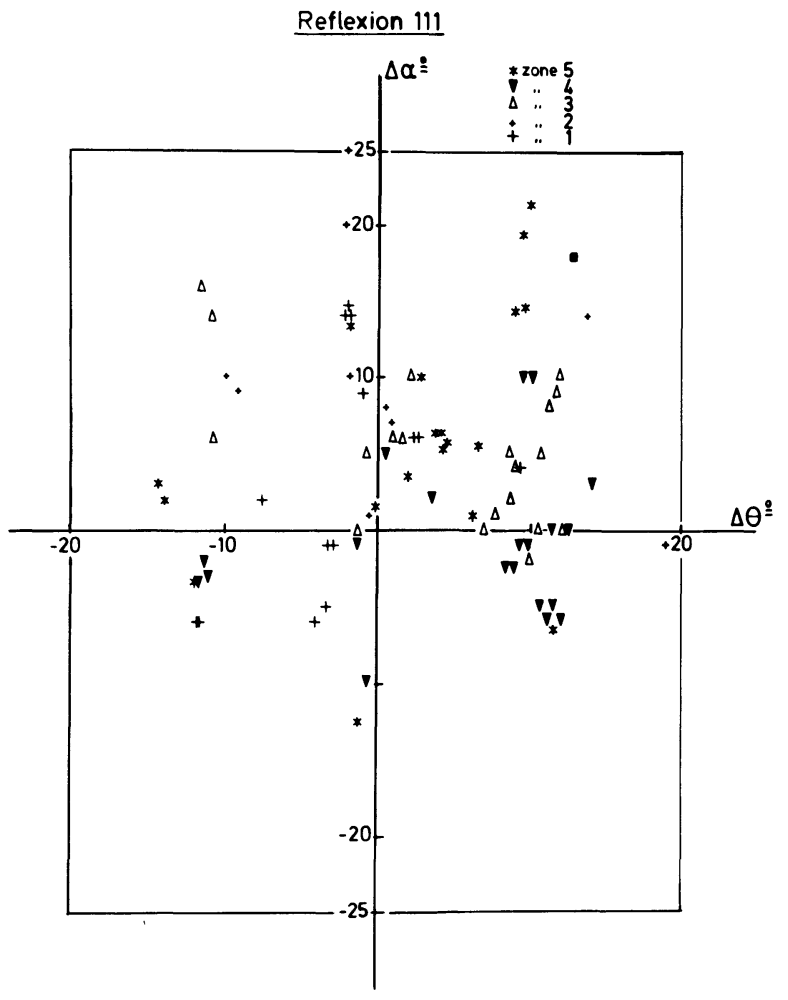

a)

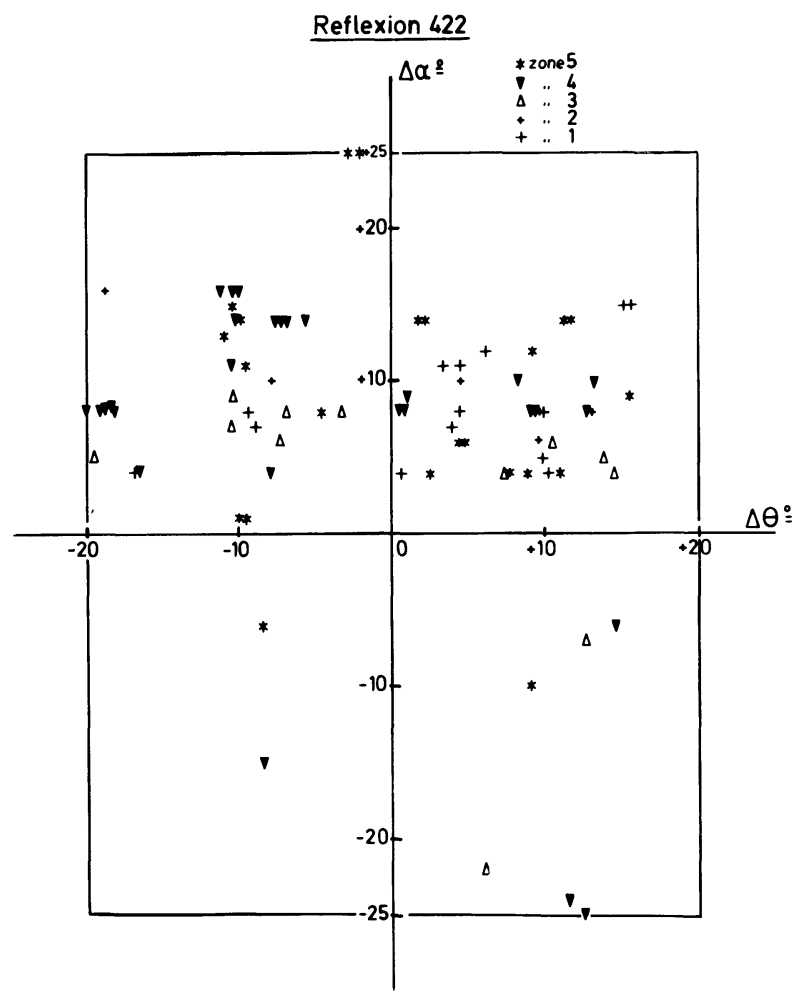

b)

Figs. $7 a$ et $b$. - Distribution des désorientations des plans (111) et (211).

[Distribution of the disorientations of the planes (111) and (211).] 
reportés tableau VII où la désorientation $\chi$ est quelconque.

Dans la croissance basaltique, la cristallisation présente une orientation privilégiée : direction [211] parallèle au gradient thermique le plus important au détriment des directions [100] et [110].

Les distributions des désorientations des plans réflecteurs par rapport à la surface des plaques sont repérées sur deux séries de diagrammes :

(i) Répartition en variations angulaires $[\Delta \alpha-\Delta \theta]$, tracés reproduits pour les plans (111) et (211). Sur les
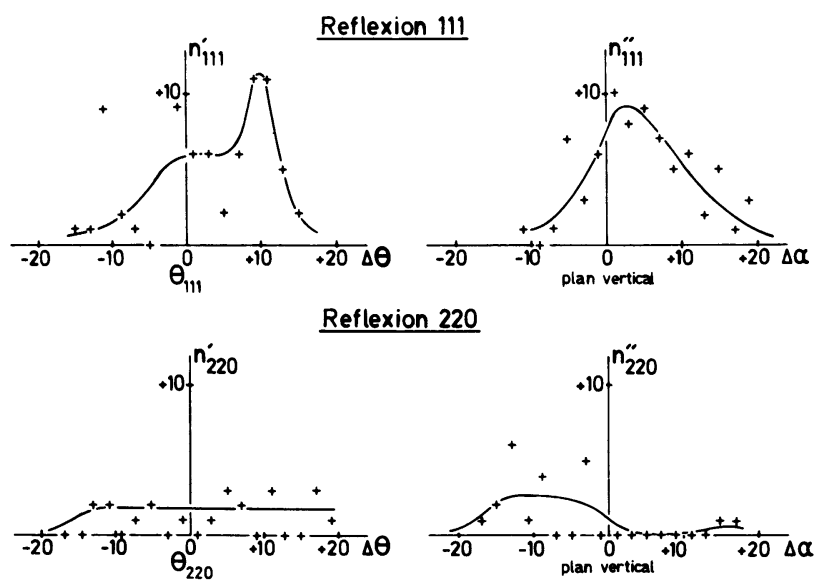

Reflexion 311
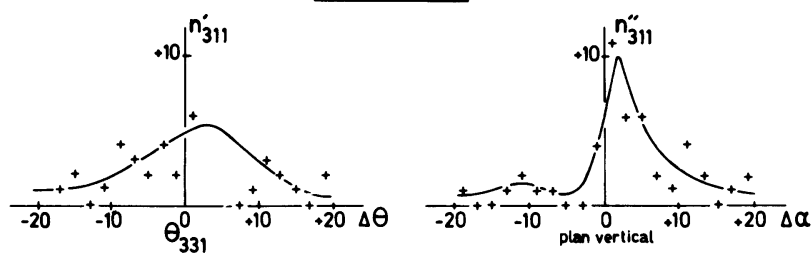

Reflexion 400
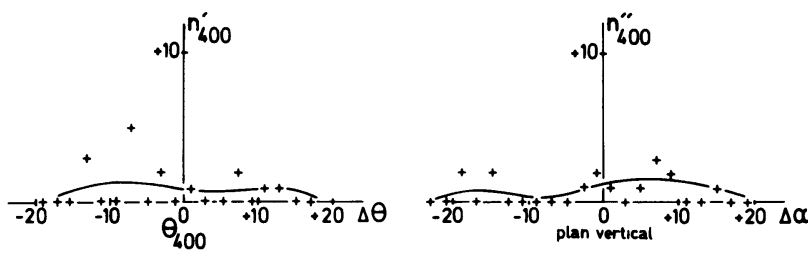

Reflexion 331
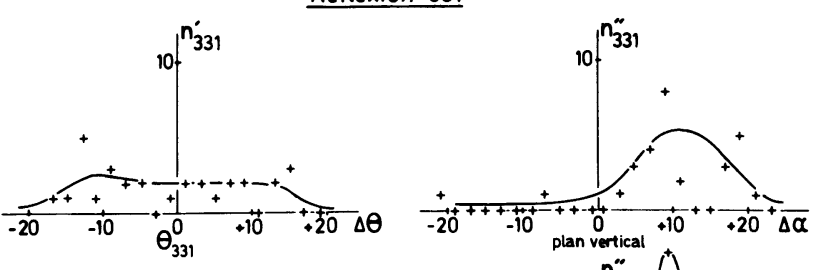

Reflexion 422
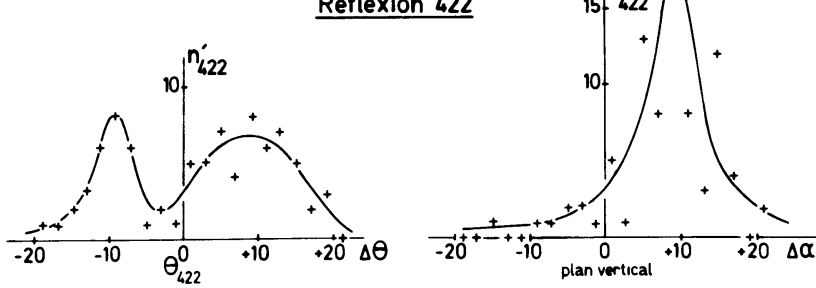

Fig. 8. - Distribution en nombre de plans réflecteurs. [Statistical distribution of the reflecting planes.] figures $a$ et $b$, les coordonnées des points sont égales aux désorientations $\Delta \alpha$ et $\Delta \theta$ d'un plan repéré par rapport à la surface de la plaque. Les plans (211) sont regroupés en majorité dans le domaine $\Delta \alpha>0$ autour de $\Delta \alpha=+8^{\circ} \pm 3^{\circ}$, la distribution en $\Delta \theta$ reste plus étalée. Il n'apparaît pas de différence en fonction de la zone explorée. Pour les plans (111) la densité des points représentatifs est supérieure dans le quadrant positif $[\Delta \alpha>0, \Delta \theta>0]$ limité à $\Delta \alpha<+10^{\circ}$ et $\Delta \theta<+12^{\circ}$, avec une distribution plus resserrée pour les zones 3 et 4 .

ii) Distribution en nombre dans des domaines de désorientations données $\Delta \theta$ et $\Delta \alpha$.

Les courbes moyennes $n_{h k l}^{\prime}=f(\Delta \theta)$ et $n_{h k l}^{\prime \prime}=f(\Delta \alpha)$ sont reportées figure 8 pour les 6 réflexions explorées. Les ordonnées sont des valeurs relatives proportionnelles à $n_{h k l}^{\prime}$ et $n_{h k l}^{\prime \prime}$;

$n_{h k l}^{\prime}=$ nombre de grains dont les plans de surface présentent par rapport à $\theta_{h k l}$ une désorientation comprise dans le domaine $\left[\Delta \theta \pm 1^{\circ}\right]$,

$n_{h k l}^{\prime \prime}=$ nombre de grains dont les plans de surface présentent par rapport à la verticale, une inclinaison comprise dans le domaine $\left[\Delta \alpha \pm 1^{\circ}\right]$. Quelle que soit la réflexion observée, les courbes indiquent une probabilité de présence non centrée sur une désorientation nulle.

Des désorientations plus ou moins importantes existent en général entre la surface des plaquettes et les plans de faibles indices des différents grains.

Ce résultat est confirmé par les diagrammes de Laue (voir Tableau VII). Il explique que la méthode d'exploration continue $(\theta-2 \theta)$ conduise à un nombre très faible de réflexions répérées.

3.4 Diffraction NeUtroniQue. - L'échantillon étudié est un cube de $8 \mathrm{~mm}$ de côté, découpé à la périphérie du bloc $\mathrm{E}_{\mathrm{IV}}$ dans la zone de cristallinite dendritique. L'axe de rotation de l'échantillon est choisi parallèle à l'axe longitudinal du lingot.

Les résultats expérimentaux sont reportés tableau $\mathrm{V}$ où $I$ est l'intensité intégrée exprimée en unité relative, $\Delta l=$ la largeur à mi-hauteur après convolution gaussienne de pics.

Les intensités cumulées, exprimées en unités relatives (8528 cps/s pour l'échantillon standard de poudre et 18390 pour l'échantillon étudié) conduisent à un rapport de normalisation de comptage égal à 2,2. La position angulaire reportée en ${ }^{\circ}$ est l'angle de la face latérale du lingot repérée par $\Downarrow$ avec le faisceau incident (Fig. 5 et Tableau I).

Bien que les pas de rotation utilisés soient trop importants pour obtenir une distribution précise des orientations cristallines, les variations des rapports $I_{\mathrm{e}} / I_{\mathrm{s}}$ des intensités diffractées par l'échantillon et par le standard pour les différentes réflexions $h k l$ enregistrées, ne sont pas dues uniquement aux désorientations des plans réflecteurs $(h k l)$ par rapport aux positions de Bragg. 
Tableau V. - Orientations privilégiées :

a) Grandeurs intervenant dans le calcul des intensités des raies

b) Résultats obtenus par diffraction des neutrons

[Preferential orientations.

a) Calculation of the intensity of the Bragg's reflections;

\begin{tabular}{|c|c|c|c|c|c|c|c|}
\hline $\begin{array}{c}\text { Réflexion } \\
h k l\end{array}$ & $\left.\frac{F_{h k l}}{f}\right|^{2}$ & $n$ & $\begin{array}{c}\frac{\sin \theta}{\lambda} \\
\lambda=1,54 \AA\end{array}$ & $\begin{array}{l}\text { rayo } \\
f_{\mathrm{Si}}\end{array}$ & $\begin{array}{l}n s X \\
L p\end{array}$ & $\begin{array}{c}\begin{array}{c}\text { neutron } \\
b=0,415 \times 10 \\
\frac{\sin \theta}{\lambda} \\
\lambda=1,27 \AA\end{array} \\
\lambda=1\end{array}$ & $\begin{array}{c}\mathrm{s}^{-12} \mathrm{~cm} \\
L\end{array}$ \\
\hline 111 & 32 & 8 & 0,132 & 11,15 & 44,9 & 0,161 & 24,4 \\
\hline 333 & 32 & 8 & 0,398 & 7,20 & 3,6 & 0,483 & 3,4 \\
\hline 220 & 64 & 12 & 0,217 & 9,30 & 15,2 & 0,263 & 9,5 \\
\hline 440 & 64 & 12 & 0,434 & 6,80 & 3,0 & 0,526 & 3,0 \\
\hline 311 & 32 & 24 & 0,254 & 8,80 & 10,5 & 0,308 & 7,1 \\
\hline 400 & 64 & 6 & 0,307 & 8,20 & 6,6 & 0,372 & 5,1 \\
\hline 331 & 32 & 24 & 0,334 & 7,80 & 5,4 & 0,405 & 4,4 \\
\hline 422 & 64 & 24 & 0,376 & 7,40 & 4,1 & 0,455 & 3,7 \\
\hline 531 & 32 & 48 & 0,454 & 6,70 & 2,1 & 0,550 & 2,9 \\
\hline
\end{tabular}

b) Results obtained by neutron diffraction.]

\begin{tabular}{|c|c|c|c|c|c|c|}
\hline Standa & $\begin{array}{l}\text { oudre } \\
\qquad \Delta l\end{array}$ & $\begin{array}{l}\text { Echantillon } \\
\text { position } \\
\text { angulaire } \\
\text { en }{ }^{\circ}\end{array}$ & $\begin{array}{c}\text { polycris } \\
I\end{array}$ & $\begin{array}{l}\text { allin } \\
\Delta l\end{array}$ & \multicolumn{2}{|c|}{$I_{\mathrm{e}} / I_{\mathrm{s}}$} \\
\hline 657 & 14 & $\begin{array}{r}90 \\
165 \\
180 \\
195 \\
255 \\
210\end{array}$ & $\begin{array}{r}8 \\
105 \\
8783 \\
296 \\
5 \\
1945\end{array}$ & $\begin{array}{r}8 \\
5 \\
625 \\
77 \\
2 \\
26\end{array}$ & $\left.\begin{array}{l}0,002 \\
0,030 \\
2,525 \\
0,085 \\
0,001 \\
1,373\end{array}\right\}$ & $\{4,01$ \\
\hline $\begin{array}{r}1648 \\
428\end{array}$ & 17 & $\begin{array}{r}90 \\
105 \\
255 \\
135 \\
240\end{array}$ & $\begin{array}{r}20 \\
8 \\
8 \\
821 \\
288\end{array}$ & $\begin{array}{r}20 \\
20 \\
4 \\
122 \\
21\end{array}$ & $\left.\begin{array}{l}0,006 \\
0,002 \\
0,002 \\
0,889 \\
0,312\end{array}\right\}$ & 1,21 \\
\hline 1195 & 21 & $\begin{array}{l}120 \\
240\end{array}$ & $\begin{array}{r}90 \\
144\end{array}$ & $\begin{array}{r}10 \\
5\end{array}$ & $\left.\begin{array}{l}0,035 \\
0,056\end{array}\right\}$ & 0,09 \\
\hline 382 & 11 & $\begin{array}{l}120 \\
165\end{array}$ & $\begin{array}{r}94 \\
206\end{array}$ & $\begin{array}{l}10 \\
98\end{array}$ & $\left.\begin{array}{l}0,114 \\
0,250\end{array}\right\}$ & 0,36 \\
\hline 677 & 22 & $\begin{array}{r}90 \\
105 \\
120 \\
240\end{array}$ & $\begin{array}{r}71 \\
1341 \\
18 \\
1285\end{array}$ & $\begin{array}{r}16 \\
26 \\
10 \\
6\end{array}$ & $\left.\begin{array}{l}0,049 \\
0,919 \\
0,012 \\
0,880\end{array}\right\}$ & 1,86 \\
\hline 1130 & 20 & $\begin{array}{r}90 \\
105 \\
165 \\
240 \\
255\end{array}$ & $\begin{array}{r}59 \\
15 \\
1453 \\
9 \\
82\end{array}$ & $\begin{array}{r}3 \\
10 \\
23 \\
6 \\
4\end{array}$ & $\left.\begin{array}{l}0,024 \\
0,006 \\
0,596 \\
0,004 \\
0,003\end{array}\right\}$ & 0,66 \\
\hline 798 & 25 & $\begin{array}{l}105 \\
120 \\
135\end{array}$ & $\begin{array}{r}7 \\
1222 \\
7\end{array}$ & $\begin{array}{l}4 \\
7 \\
4\end{array}$ & $\left.\begin{array}{l}0,004 \\
0,710 \\
0,004\end{array}\right\}$ & 0,71 \\
\hline
\end{tabular}

Des orientations privilégiées apparaissent :

- Croissance suivant des plans (111) parallèles à l'axe du lingot. Dans le plan médian, perpendiculaire à l'axe de tirage, les désorientations entre plans (111) restent $\leqslant 15^{\circ}$ et leur orientation moyenne fait un angle $\simeq 12^{\circ}$ avec la face latérale du lingot (voir Fig. 9).

- Croissance suivant des plans (110) et (331) parallèles à l'axe du lingot.

- Les autres réflexions enregistrées ont des intensités inférieures à celles des raies du standard.

- Les désorientations entre plans repérés (Fig. 9) ne correspondent pas exactement aux angles interplanaires du système cubique mais les écarts restent inférieurs à $\pm 20^{\circ}$, ce qui semble bien confirmer des orientations de croissance privilégiée.
- L'incertitude de l'orientation des plans réflecteurs par rapport aux plans verticaux bissecteurs n'a pas été évaluée. Elle est au moins égale à l'ouverture angulaire verticale du détecteur, soit $\cong 3,6^{\circ}$.

Ces premiers résultats indiquent que les orientations de croissance privilégiées sont différentes dans les zones à croissance dendritique et à croissance colonnaire. Ils sont incomplets et doivent être confirmés par des mesures de texture en diffraction neutronique. Ces études sont en cours.

Des expériences semblables, faites sur le même échantillon par diffraction des rayons $\mathrm{X}$ en réflexion, avaient conduit à une statistique de comptage trop faible bien que la durée de l'expérience ait été supérieure (mesures non reportées dans le tableau V).

Ces expériences sommaires ont montré la supériorité 


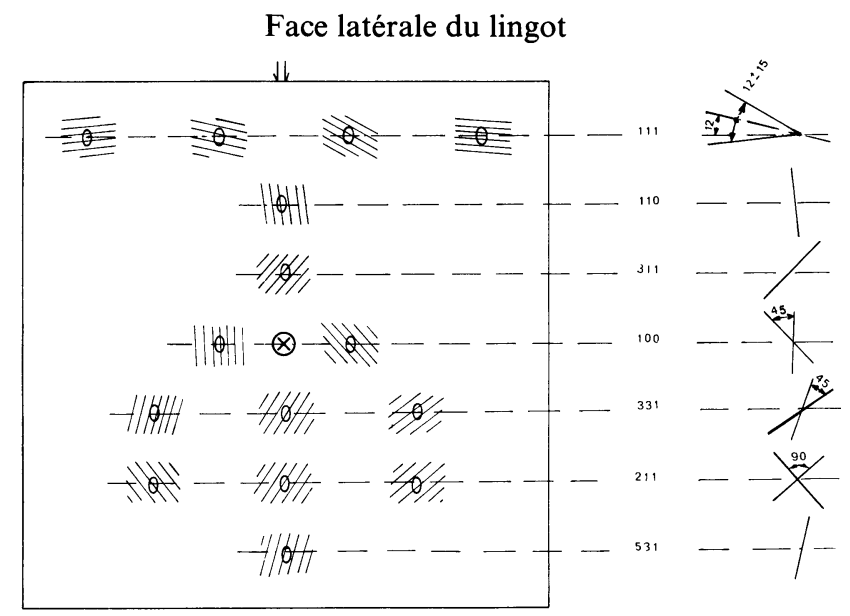

Fig. 9. - Traces des plans réflecteurs dans le plan médian de l'échantillon perpendiculaire à l'axe de tirage.

L'axe du lingot ou axe de tirage est parallèle à l'axe de rotation $\otimes$.

[Traces of the reflecting planes in the median plane of the sample, perpendicular to the crucible axis.]

de la diffraction neutronique sur la diffraction des rayons $X$ pour l'étude d'un tel matériau. Plus qu'à l'invariance du facteur $b$ en fonction de l'angle $\theta$, cette supériorité est due à la fois au volume très supérieur du matériau exploré (taille du faisceau incident neutronique dans un rapport $\geqslant 5$ à celle du faisceau de rayons $\mathrm{X}$; absorption des neutrons négligeable par $\mathrm{Si}$ ) et à la possibilité d'enregistrer simultanément les intensités diffractées dans un domaine angulaire important (montage D1B équipé d'un multidétecteur).

Les analyses précédentes sont obtenues à partir de méthodes précises mais longues et délicates qui ne conviennent pas dans le milieu industriel pour caractériser le matériau au cours des mises au point d'élaboration. Une méthode moins précise mais très rapide, ne présentant pas de difficulté particulière et de coût très faible a été développée.

4. Contrôle rapide de l'orientation à partir de l'observation des couleurs d'interférence des lames minces. 4.1 Principe. - Ces lames minces sont formées par attaque électrochimique du silicium polycristallin utilisé comme anode dans une cellule d'électrolyse classique (électrode de travail : silicium, électrode de référence au calomel saturé, électrode intermédiaire grille de platine). Après électrolyse, faite dans des conditions bien définies, la surface attaquée est recouverte d'une couche dont les couleurs variées, observées en lumière blanche sous incidence normale, différencient très nettement les cristallites les uns des autres (photo $\mathrm{n}^{\mathrm{O}} 3$ ).

L'attaque électrolytique des semiconducteurs a été étudiée par de nombreux auteurs et les mécanismes de dissolution anodique du silicium dans les solutions hydrofluorhydriques décrits. Des désaccords sub- sistent sur la composition des dépôts [15-19]. Nous rappelons seulement que pour conserver le dépôt formé à la surface de l'anode, l'attaque doit se faire en dehors du domaine d'électropolissage, c'est-à-dire

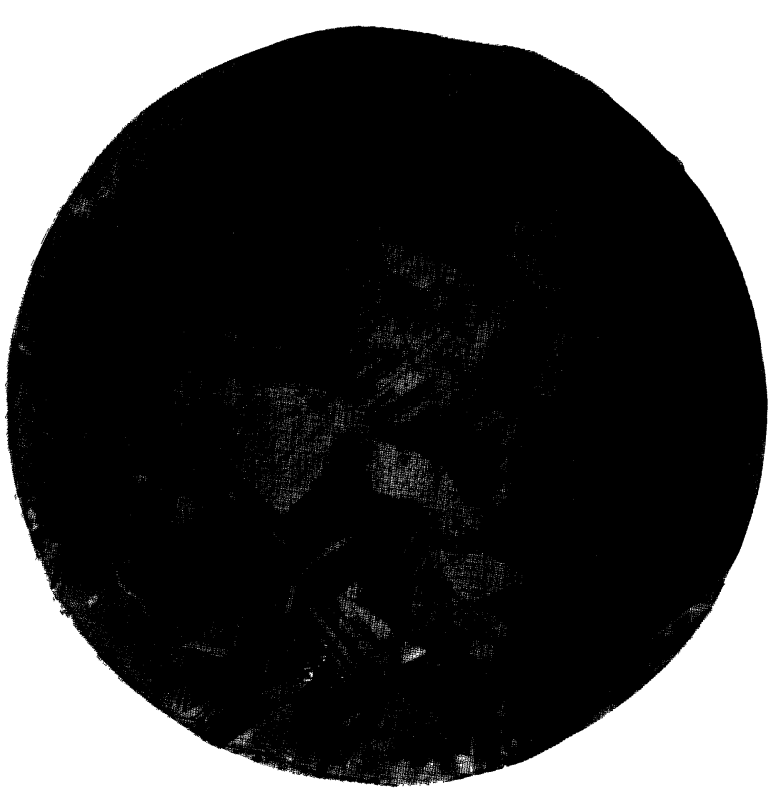

a)

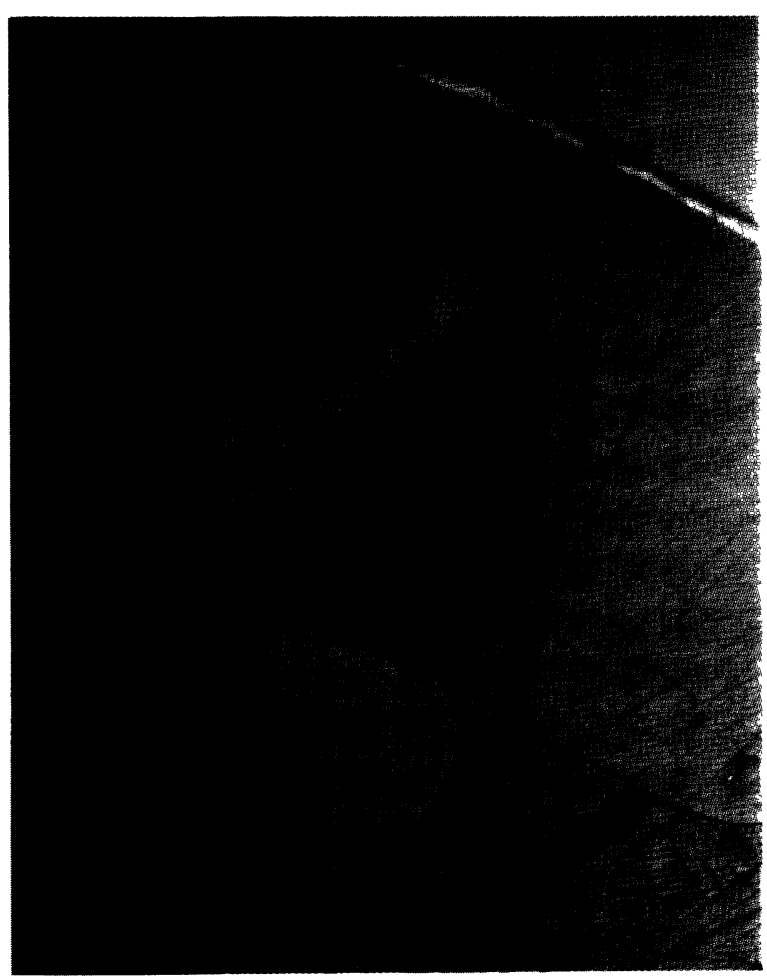

b)

Photo 3. - Aspect de la plaquette de silicium polycristallin après attaque électrochimique.

[Aspect of the polycrystalline silicon wafer after electrolytical etching.] 
sous une faible densité de courant, inférieure à la valeur critique pour laquelle la couche se détache de l'électrode. De plus, la concentration de l'électrolyte en acide fluorhydrique doit rester faible.

Dans ces conditions, le silicium serait dissous à l'état divalent; il y aurait formation d'une couche de silicium poreux microcristallin ou amorphe recouvert d'un film d'oxyde. En solution diluée, les molécules HF sont en nombre insuffisant au voisinage de l'électrode pour que l'oxyde soit réduit. Cet oxyde, insoluble dans l'eau, forme un film résistant sur la surface anodique. D'indice de réfraction très différent de celui du silicium, ce film jouerait le rôle de lame mince dont l'épaisseur dépendrait du grain attaqué.

Des études systématiques ont permis d'optimiser les conditions expérimentales (travail reporté Réfs. $[20,21,22])$ :

( anode : plaque de silicium polycristallin $\varnothing=20 \mathrm{~mm}$, électrolyte :

solution aqueuse 50/50 éthanol $+\mathrm{HF} 5 \%$,

tension de polarisation $9 \pm 0,1 \mathrm{~V}$,

densité de courant $J \lesssim 40 \pm 4 \mathrm{~mA} / \mathrm{cm}^{2}$,

durée de l'électrolyse : $25 \pm 0,20 \mathrm{~min}$.

Des corrélations entre la vitesse d'attaque (ou épaisseur du dépôt) et les divers paramètres régissant l'électrolyse ont été établis $[4,5]$.

Dans le domaine de faible densité de courant utilisé ici, les incertitudes sur l'épaisseur du dépôt restent faibles et proportionnelles aux incertitudes des différents paramètres, soit :

- durée de l'électrolyse pour $\Delta t / t \cong 1 \%$ autour de $25 \min \Delta e / e \cong 1 \%$,

- concentration de l'électrolyte autour de la concentration $\eta_{\mathrm{HF}}=5 \%$

pour $\Delta \eta_{\mathrm{HF}} / \eta_{\mathrm{HF}} \cong 5 \% \Delta e / e \cong 2 \%$,

- température autour de $T=22{ }^{\circ} \mathrm{C}$

pour $\Delta T \cong \pm 1{ }^{\circ} \mathrm{C} \Delta e / e \cong 1 \%$,

- illumination : son influence sur Si type $\mathrm{P}$ est faible et les conditions d'éclairement suffisamment reproductibles pour négliger cette erreur,

- densité de courant pour une variation $\Delta J / J \cong 10 \%$

$$
\left\{\begin{array}{c}
\Delta e / e \cong 3 \% \text { pour silicium type } \mathrm{P} \text { résistivité } \\
\cong 0,01 \Omega-\mathrm{cm} \\
\Delta e / e \cong 5 \% \text { pour silicium type résistivité }
\end{array}\right.
$$

$$
\cong 1,5 \Omega-\mathrm{cm}
$$

- concentration des impuretés ou dopant à $J \cong 30 \mathrm{~mA} / \mathrm{cm}^{2}$

variation en donneurs $10^{15}$ à $10^{6}$ atomes $/ \mathrm{cm}^{3}$

$$
\frac{\Delta e}{e} \cong 10 \%
$$

variation en accepteurs $10^{15}$ à $10^{16}$ atomes $/ \mathrm{cm}^{3}$

$$
\frac{\Delta e}{e} \cong 8 \% \text {. }
$$

Les incertitudes sur les paramètres régissant l'électrolyse jouent un rôle relativement faible $(<5 \%)$ sur l'imprécision de l'épaisseur du dépôt. Il n'en est pas de même de l'influence des concentrations en impuretés ( $\cong 10 \%$ et plus). Cette influence est la principale limite de la validité de la méthode.

Si le taux d'impuretés est suffisamment faible pour que la.densité des porteurs de charge soit peu modifiée par leur présence ou si ces impuretés sont réparties de façon homogène dans le semiconducteur, une fois les paramètres de l'électrolyse adoptés et bien contrôlés, l'épaisseur du dépôt formé à la surface de l'anode ne dépend que de la densité réticulaire des plans de surface donc de l'orientation cristalline des grains.

Après l'étude du dépôt électrolytique, l'orientation cristalline a été déterminée pour un grand nombre de grains à l'aide de diagrammes de Laue. Des corrélations ont pu être établies entre épaisseur de la couche d'oxyde et orientation des plans attaqués, et les limites de validité de la méthode précisées.

Seule l'étude liée directement à l'orientation cristalline est reportée ici. L'attaque anodique est de plus une méthode simple et rapide pour caractériser les défauts de surface du silicium et remplace, avec une résolution meilleure, les attaques chimiques suivies d'analyses en E.B.I.C. généralement adoptées pour l'étude des semiconducteurs. Ces avantages ont déjà été décrits dans des travaux [20 à 23].

4.2 ETUdE DU DÉPOT ÉlECTROLyTIQUE. 4.2.1 Epaisseur de la couche. - L'épaisseur de la couche a été mesurée optiquement à l'aide d'un microscope $(G \times 200)$ équipé d'un interféromètre de Nomarsky. Après étalonnage de l'interferomètre en lumière monochromatique $(\lambda=5960 \AA)$, le décalage des franges colorées observées en lumière blanche, à la frontière de la couche en différents domaines de couleurs variées, permet d'évaluer les épaisseurs du dépôt sur plusieurs grains avec une précision atteignant $5 \%$ lorsque la différence de marche est nette.

Ces déterminations sont difficiles et imprécises lorsque les variations d'épaisseur se font progressivement (joint de grains, bords des cratères de pulvérisation, etc...). Dans ce cas, les mesures se font à l'aide d'un microrugosimètre à palpeur mécanique (alphastep) avec une précision de $\pm 1 \%$.

L'épaisseur des dépôts électrolytiques varie de 13500 à $20000 \AA$ alors que les teintes observées par réflexion, en incidence normale, recouvrent la totalité du second ordre et une partie des premier et troisième ordres de l'échelle des teintes de Newton (soit $4000 \AA<\delta=2 n e<15000 \AA$ ).

Il y a donc un important décalage des franges. Ce déplacement s'explique si on admet le dépôt constitué d'une couche d'oxyde dans sa partie supérieure, suivie d'une couche intermédiaire de matériau homogène d'indice de réfraction élevé, voisin de celui du substrat, tel que $\mathrm{Si}$ amorphe ou cristallin $\left[n\left(\mathrm{SiO}_{2}\right)=1,55\right.$; $n(\mathrm{Si}$ cristallin $)=3,85 ; n(\mathrm{Si}$ poreux $) \cong n(\mathrm{Si}$ amorphe $)$ $=3,83$; dans le spectre visible, [24 à 26]. Seule la partie 
supérieure du dépôt interviendrait dans l'épaisseur de la lame mince optique. Cette hypothèse est confirmée par les variations d'épaisseur $\Delta e$ mesurées au rugosimètre au passage du palpeur sur des grains adjacents de couleur contrastées. La différence de marche optique $\Delta \delta=2 n \Delta e$ reste toujours en bon accord avec les écarts reportés sur l'échelle des teintes de Newton [27] si $n$ reste voisin de 1,50.

Exemple :

$$
\begin{aligned}
& \text { ( } \Delta e=1600 \pm 50 \AA \text { passage d'un grain vert ( } 2^{\mathrm{e}} \text { ordre) } \\
& \left\{\Delta e=1300 \pm 50 \AA \begin{array}{l}
\text { à un grain jaune }(1 \text { er ordre }) \\
\text { passage d'un grain orange }
\end{array}\right. \\
& \left\{\begin{array}{r}
\Delta e=1300 \pm 50 \AA \\
\left(2^{\mathrm{e}}\right. \text { ordre) à un grain pourpre, }
\end{array}\right. \\
& \text { teinte sensible, séparation } 1^{\mathrm{er}} \text { et } \\
& 2^{\mathrm{e}} \text { ordres. }
\end{aligned}
$$

L'étude de la composition de la couche a donc été entreprise pour vérifier cette hypothèse.

4.2.2 Analyse de la couche. - Le dépôt électrolytique a été analysé par diffraction des rayons $\mathrm{X}$ et par analyse ionique.

1) Diffraction des rayons $\mathrm{X}$. - Les diagrammes $(\theta-2 \theta)$ en enregistrement continu ont été obtenus sur le goniomètre Philips (montage par réflexion).

Bien que l'épaisseur du dépôt soit faible, la surface importante irradiée permet de mettre en évidence la présence d'oxydes de silicium :

- apparition des réflexions les plus intenses de la cristobalite $\alpha$ (système tétragonal),

- enregistrement d'anneaux ne correspondant ni au silicium amorphe [28] ni à la silice amorphe [29]. L'oxyde de silicium cristallisé sous forme de tridymite (système pseudohexagonal) présente un polytypisme marqué qui entraîne une variation importante de la largeur des raies. Un grand nombre de ses réflexions rapprochées peuvent, si elles sont élargies, se superposer et apparaître comme des anneaux. Leur position moyenne est en bon accord avec les enregistrements tracés,

— des réflexions élargies correspondent à du silicium microcristallisé. L'épaisseur des couches déposées reste toujours très inférieure à "l'épaisseur infinie " correspondant à $\lambda \mathrm{CuK}_{\alpha}$. Les rayons $\mathrm{X}$ traversent entièrement la couche et les raies de Bragg du silicium polycristallin du substrat d'intensité bien supérieure, sont également enregistrées lorsque l'orientation des plans de surface le permet.

Aucune autre réflexion n'est mise en évidence.

2) Analyse ionique. - i) Dispositif expérimental. L'analyseur ionique "CAMECA S.M.I. 300 » a été utilisé $\left({ }^{2}\right)$.

L'échantillon maintenu sous vide $\left(2 \times 10^{-17}\right.$ torr $)$ est bombardée par un faisceau focalisé d'ions primaires d'argon $\mathrm{A}^{+}$. La surface attaquée $\left(\cong 0,5 \times 0,5 \mathrm{~mm}^{2}\right)$

( $\left.{ }^{2}\right)$ Mesures faites au Laboratoire du C.N.R.S. de Bellevue, en collaboration avec Mme Barbezat. est assez petite pour être contenue dans un cristallite isolé.

Par bombardements successifs, les couches atomiques de plus en plus profondes sont pulvérisées, l'enregistrement des spectres correspondant à chaque bombardement conduit à une analyse de la composition de la couche.

Les particules émises peuvent être neutres ou ionisées $(+$ ou -$)$. Dans le cas d'échantillons isolants, les ions positifs sont plus faciles à obtenir. L'analyse est faite en courant d'émission positif pour les différents éléments observés à l'exception du fluor de faible pouvoir émissif et dont la sensibilité est supérieure en courant négatif.

L'échantillon est porté à une tension de $4500 \mathrm{~V}$ et les particules ionisées accélérées dans un champ électrique. Dans nos expériences, une tension de filtrage égale à $22 \mathrm{~V}$, appliquée entre le miroir électrosstatique et l'échantillon permet d'obtenir des raies plus fines sur les spectres.

Un phénomène de charge apparaît sur les échantillons recouverts de la couche d'oxyde isolante après électrolyse, phénomène non observé sur le silicium non attaqué. Il a été nécessaire de déposer sur les surfaces oxydées une mince couche métallique pour permettre l'écoulement des charges produites par les ions primaires. La couche d'or évaporée $(200 \AA)$ est pulvérisée sur la surface du cratère laissant un passage libre pour les ions primaires incidents et les ions secondaires émis, le cadre conducteur subsistant est suffisant pour permettre l'écoulement des charges.

Les analyses se font :

- soit en explorant un domaine restreint autour de deux éléments choisis : cycles rapides de $24 \mathrm{~s}$ comprenant deux balayages centrés sur les masses $M_{1} \pm 0,15$ et $M_{2} \pm 0,15$ et tracé du profil des deux éléments considérés :

$\left\{\right.$ ions monoatomiques monochargés $160^{+}$

$\left\{\right.$ ions polyatomiques monochargés $18 \mathrm{H}_{2} \mathrm{O}^{+}$

$\left\{\right.$ ions monoatomiques monochargés $16 \mathrm{O}^{+}$

ions monoatomiques monochargés $30 \mathrm{Si}^{+}$

$\left\{\right.$ ions monoatomiques monochargés $16 \mathrm{O}^{-}$

ions monoatomiques monochargés $19 \mathrm{~F}^{-}$

- soit en enregistrant toutes les masses de 1 à 150 par une variation continue de l'induction magnétique. La durée de balayage d'un tel spectre est voisine de $200^{\prime \prime}$ (cette dernière analyse est faite en courant positif).

ii) Vitesses de pulvérisation, épaisseur de couches successives. - Nous avons tout d'abord vérifié que la vitesse de pulvérisation était sensiblement proportionnelle à la densité du bombardement et constante en fonction du temps lorsque la composition ne varie pas. A partir de mesures faites sur des monocristaux de silicium plan de surface (331), l'épaisseur de la couche d'oxygène adsorbée " $e_{\mathrm{O}_{\mathrm{a}}}$ " a été déterminée ainsi que les vitesses moyennes de pulvérisation de l'oxygène 


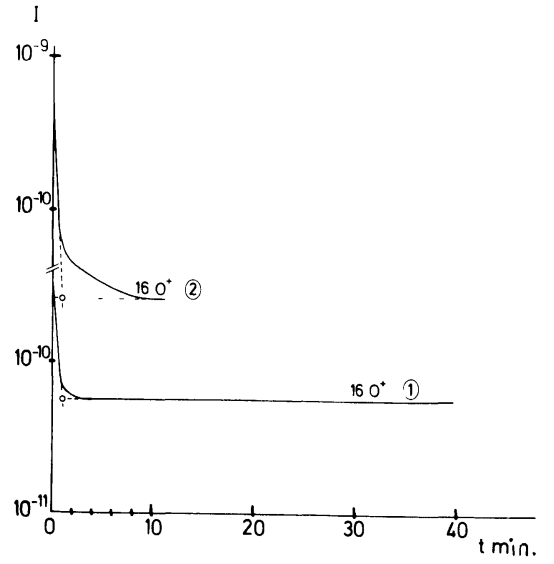

Fig. 10. - Profils d'oxygène $I=f(t)$

\begin{tabular}{l|c|c|c} 
& Spectre & I & II \\
\cline { 2 - 4 } $\begin{array}{c}\text { Densité de } \\
\text { bombardement }\end{array}$ & $I_{p}$ & $37 \mathrm{nA}$ & $45 \mathrm{nA}$ \\
$\begin{array}{c}\text { Durée de } \\
\text { pulvérisation }\end{array}$ & $t \mathrm{~s}$ & 100 cycles de $24^{\prime \prime}$ & 27 cycles de 24" \\
$\begin{array}{c}\text { Profondeur du } \\
\text { cratère }\end{array}$ & $e$ & $3450 \AA$ & $1100 \AA$
\end{tabular}

Echantillon observé : monocristal-plan de surface (331). $I$ exprimée en unités relatives.

[Oxygen profiles $I=f(t)$

\begin{tabular}{|c|c|c|c|}
\hline 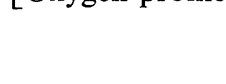 & $\begin{array}{l}\text { Spec- } \\
\text { trum }\end{array}$ & I & II \\
\hline $\begin{array}{l}\text { Bombardment } \\
\text { density }\end{array}$ & $I_{p}$ & $37 \mathrm{nA}$ & $45 \mathrm{nA}$ \\
\hline Sputtering time & $t \mathrm{~s}$ & 100 cycles of $24^{\prime \prime}$ & 27 cycles of $24^{\prime \prime}$ \\
\hline Crater depth & $e$ & $3450 \AA$ & $1100 \AA$ \\
\hline
\end{tabular}

Sample : monocrystal-plane (331). I expressed in relative units.]

adsorbé et du silicium cristallisé $" v_{\mathrm{O}_{\mathrm{a}}}$ " et $" v_{\mathrm{Si}_{\mathrm{c}}}$ ". Les contrôles sur les plaquettes polycristallines (C.G.E.E.I.) ont montré que la couche d'oxygène adsorbée restait sensiblement de même épaisseur sur les diffé-

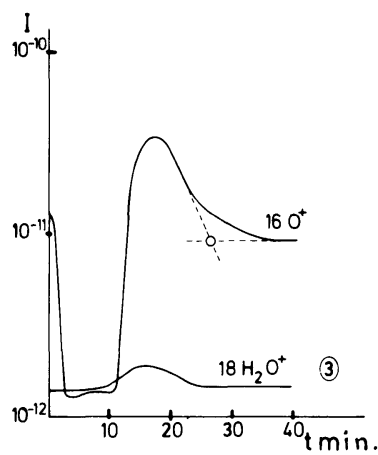

Fig. 11. - Profils d'oxygène $16 \mathrm{O}^{+}$et d'eau $18 \mathrm{H}_{2} \mathrm{O}^{+}-$ spectre III.

[Profiles $16 \mathrm{O}^{+}$and $18 \mathrm{H}_{2} \mathrm{O}^{+}-$spectrum III.] rents échantillons et que " $v_{\mathrm{Si}_{\mathrm{c}}}$ " était conservée sur les grains du silicium polycristallin :

$$
\left\{\begin{array}{l}
\left\langle e_{\mathrm{O}_{\mathrm{a}}} » \cong 50 \text { à } 60 \AA,\right. \\
\left\langle v_{\mathrm{O}_{\mathrm{a}}} » \cong 0,02 \pm 0,005 \AA / \mathrm{s} / \mathrm{nA},\right. \\
\left\langle v_{\mathrm{Si}_{\mathrm{c}}} » \cong 0,04 \pm 0,005 \AA / \mathrm{s} / \mathrm{nA} .\right.
\end{array}\right.
$$

Ces résultats sont obtenus à partir des profils tels que ceux représentés figure 10. A partir de l'allure des profils tracés (Figs. 11 à 15), des profondeurs des cratères creusés après bombardement et des épaisseurs des dépôts électrolytiques, on obtient un système d'équations en nombre suffisant pour déterminer les vitesses de pulvérisation des couches successives et du substrat ainsi que leurs épaisseurs approximatives :

vitesse de pulvérisation du dépôt d'or

$$
v_{\text {or }} \cong 0,01 \AA / \mathrm{s} / \mathrm{nA}
$$

vitesse de pulvérisation de la couche d'oxyde

$$
v_{\mathrm{Si}_{n} \mathrm{O}_{p}} \cong 0,15 \AA / \mathrm{s} / \mathrm{nA}
$$

vitesse de pulvérisation du silicium poreux du dépôt

$$
v_{\text {Sip }} \cong 0,04 \AA / \mathrm{s} / \mathrm{nA}
$$

vitesse de pulvérisation du silicium cristallin du substrat

$$
v_{\mathrm{Si}_{\mathrm{c}}} \cong 0,03 \AA / \mathrm{s} / \mathrm{nA} .
$$

Les études ont porté sur plusieurs cristallites de coloration différente (exemples de résultats obtenus reportés dans le tableau VI).

Les profils tracés confirment l'existence d'une couche d'oxyde suivie d'une couche de silicium poreux contenant de l'oxygène et du fluor (ce dernier en faible quantité) dont les taux diminuent progressivement jusqu'au substrat.

Les épaisseurs des couches d'oxyde sont en bon accord avec les résultats optiques obtenus et confirment que seul l'oxyde $\mathrm{Si}_{n} \mathrm{O}_{p}$ joue le rôle de couche mince,

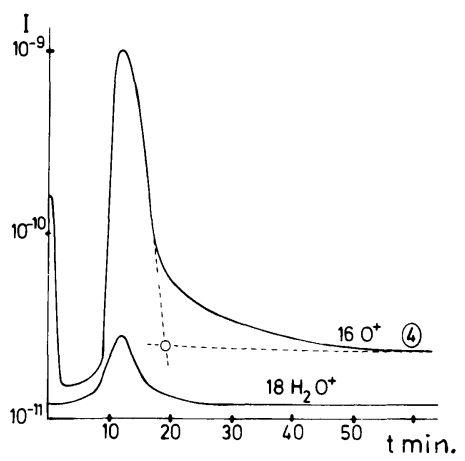

Fig. 12. - Profils d'oxygène $16 \mathrm{O}^{+}$et d'eau $18 \mathrm{H}_{2} \mathrm{O}^{+}-$ spectre IV.

[Profiles $16 \mathrm{O}^{+}$and $18 \mathrm{H}_{2} \mathrm{O}^{+}-$spectrum IV.] 


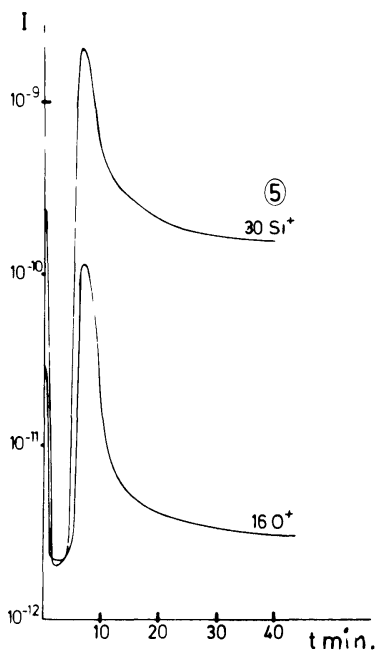

Fig. 13. - Profils d'oxygène $16 \mathrm{O}^{+}$et de silicium $30 \mathrm{Si}^{+}-$ spectre $\mathrm{V}$.

[Profiles $16 \mathrm{O}^{+}$and $30 \mathrm{Si}^{+}$- spectrum V.]

sa composition doit rester telle que l'indice de réfraction $n$ soit voisin de 1,5. Le fluor a été mis en évidence mais avec un taux de présence faible (Fig. 15). On peut admettre que dans la couche oxydée et dans la couche intermédiaire, sa présence à l'état gazeux (soit sous forme de $\mathrm{SiF}_{4}$ ou $\mathrm{Si}_{2} \mathrm{~F}_{6}$ d'indice $n \cong 1-[30]$ ne modifie pas les caractéristiques optiques de la couche.

Remarque. - La comparaison des figures 13 et 15 montre la sensibilité supérieure en émission positive pour les ions oxygène : $v\left(\mathrm{Si}_{n} \mathrm{O}_{p}\right)^{-} \sim 1 / 2 v\left(\mathrm{Si}_{n} \mathrm{O}_{p}\right)^{+}$.

iii) Constitution des couches. -- Pour analyser la couche déposée, nous avons suivi l'évolution de l'ion

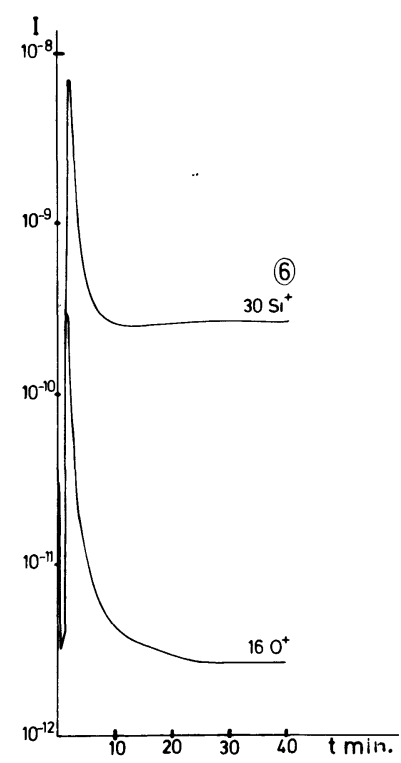

Fig. 14. - Profils d'oxygène $16 \mathrm{O}^{+}$et de silicium $30 \mathrm{Si}^{+}-$ spectre VI.

[Profiles $16 \mathrm{O}^{+}$and $30 \mathrm{Si}^{+}-$spectrum VI.]

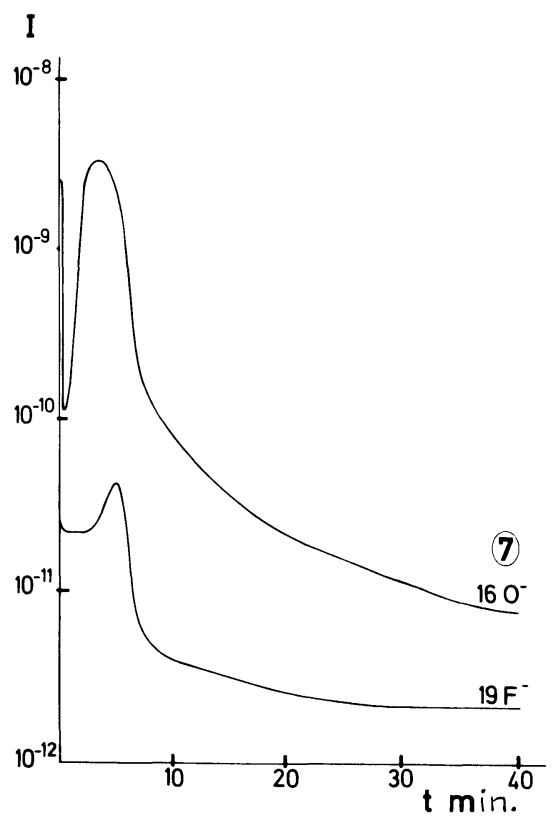

Fig. 15. - Profils d'oxygène $16 \mathrm{O}^{-}$et de fluor $19 \mathrm{~F}^{-}-$ spectre VII.

[Profiles $16 \mathrm{O}^{-}$and $19 \mathrm{~F}^{-}$- spectrum VII.]

${ }^{28} \mathrm{Si}$ et celle des ions moléculaires que forme le silicium avec l'hydrogène et l'oxygène. La figure 16 indique très nettement la faible teneur en $\mathrm{O}$ et $\mathrm{H}$ en dehors de la couche d'oxyde. On peut donc bien admettre que le silicium poreux se comporte optiquement comme le substrat et ne faire intervenir que $\mathrm{Si}_{n} \mathrm{O}_{p}$ dans l'épaisseur optique de la lame mince [nous rappelons que les indices de réfraction des différents oxydes restent compris entre 1,4 et 1,7 dans le spectre visible [24]].

Remarque. - Le pouvoir émissif des ions est très dépendant des associations considérées et de la matrice d'où ils sont extraits. Ainsi l'intensité correspondant à ${ }^{28} \mathrm{Si}$ est plus importante dans la couche d'oxyde que dans la couche poreuse ou le substrat. De même, le pouvoir émissif des ions polyatomiques $\mathrm{SiO}$ est très supérieur à celui des ions $\mathrm{Si}_{2} \mathrm{O}$ sans que cela indique une concentration en $\mathrm{SiO}$ supérieure à celle de $\mathrm{Si}_{2} \mathrm{O}$ dans la couche.

La comparaison des groupements polyatomiques observés dans les dépôts électrolytiques avec ceux obtenus pour le quartz $\mathrm{SiO}_{2}$ [31], indique que la couche est principalement constituée de $\mathrm{SiO}_{2}$ (voir Figs. $17 a$ et $17 b$ ). Lorsque des couches plus profondes sont bombardées les rapports $\mathrm{Si} / \mathrm{Si}_{n} \mathrm{O}_{p}$ augmentent quel que soit l'oxyde considéré puis se stabilisent lorsque le substrat est atteint $(\mathrm{Si} / \mathrm{SiO} \cong 60$; $\mathrm{Si} / \mathrm{SiO}_{2} \cong 1800$ ).

De même l'évolution des séries $\mathrm{SiO}_{p}$ et $\mathrm{Si}_{2} \mathrm{O}_{p}$ en fonction de la durée du bombardement indique que la composition de la couche oxydée reste voisine de celle du quartz (Figs. $18 a$ et $b$ ).

La variation de composition de la couche peut également être contrôlée à partir de l'évolution de la 
Tableau VI. - Epaisseur des couches successives dans le dépôt électrolytique

[Thickness of the successive layers in the electrochemical depositing.]

\begin{tabular}{|c|c|c|c|c|c|}
\hline Spectres. Figures & $\mathrm{III}_{2}$ & $\mathrm{IV}_{3}$ & $\mathrm{~V}_{4}$ & $\mathrm{VI}_{5}$ & $\mathrm{VII}_{6}$ \\
\hline $\begin{array}{l}\text { Profils tracés } \\
\text { Densité de bombardement en } \mathrm{nA} \\
\text { Durée du bombardement } \\
\text { en seconde } \\
\text { Profondeur du cratère en } \AA \\
\text { Echantillon observé } \\
\text { Coloration du cristallite } \\
\text { Epaisseurs approximatives des } \\
\text { couches successives en } \AA \\
\text { Oxygène adsorbé } \\
\text { Or déposé } \\
\mathrm{Si}_{n} \mathrm{O}_{p} \\
\mathrm{Si} \text { poreux } \\
\mathrm{Si} \text { substrat } \\
\text { Différence de marche optique } \\
\text { dańs la couche } \mathrm{Si}_{n} \mathrm{O}_{p} \\
\delta=2 \text { ne cos } \varnothing \text { avec } n \simeq 1,55 \\
\text { pour } \mathrm{SiO}_{2}, \cos \varnothing \cong 1\end{array}$ & $\begin{array}{c}16 \mathrm{O}^{+} \text {et } 18 \mathrm{H}_{2} \mathrm{O} \\
28 \\
2400 \\
3950 \\
\mathrm{E}_{1} \\
\text { jaune verdâtre } \\
2^{\mathrm{e}} \text { ordre } \\
\\
\simeq 60 \\
200 \\
\simeq 2900 \\
\simeq \quad 840 \\
0 \\
\end{array}$ & $\begin{array}{c}16 \mathrm{O}^{+} \text {et } 18 \mathrm{H}_{2} \mathrm{O}^{+} \\
47 \\
3720 \\
7800 \\
\mathrm{E}_{\mathrm{I}} \\
\text { violet indigo } \\
\text { 3e ordre }\end{array}$ & $\begin{array}{c}16 \mathrm{O}^{+} \text {et } 30 \mathrm{Si}^{+} \\
76 \\
2400 \\
8000 \\
\mathrm{E}_{\mathrm{I}} \\
\text { orangé } \\
2^{\mathrm{e}} \text { ordre }\end{array}$ & $\begin{array}{c}16 \mathrm{O}^{+} \text {et } 30 \mathrm{Si}^{+} \\
245 \\
2400 \\
23400 \\
\mathrm{E}_{\mathrm{I}} \\
\text { jaune verdâtre } \\
3^{\mathrm{e}} \text { ordre }\end{array}$ & $\begin{array}{c}16 \mathrm{O}^{-} \text {et } 19 \mathrm{~F}^{-} \\
80 \\
4800 \\
10100 \\
\mathrm{E}_{\mathrm{I}} \\
\text { jaune pur } \\
2^{\mathrm{e}} \text { ordre }\end{array}$ \\
\hline
\end{tabular}

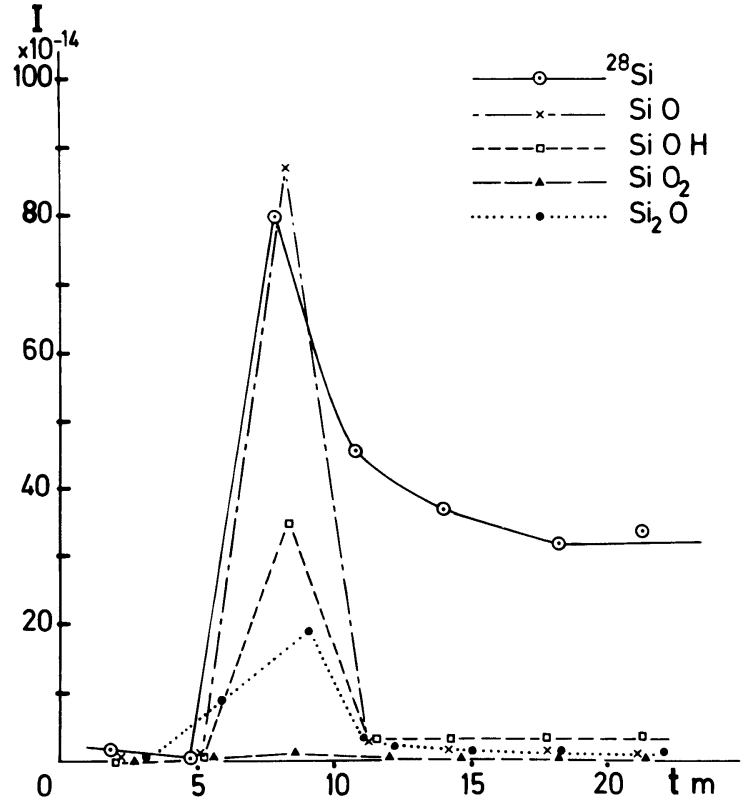

Fig. 16. - Profils du silicium et de ses composés avec l'oxygène et l'hydrogène.

[Profiles of silicon and its compounds with oxygen and hydrogen.]

concentration isotopique d'un élément. Les enregistrements obtenus à partir de spectres de bombardements successifs effectués sur un même cratère ont mis en évidence un effet isotopique d'émission pour le silicium. Ce phénomène avait déjà été observé et en partie expliqué [32]. Les rendements d'ionisation sont différents d'un isotope à l'autre. Pour deux isotopes de

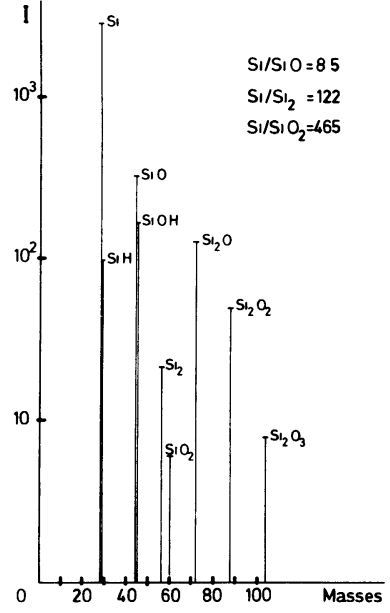

a)

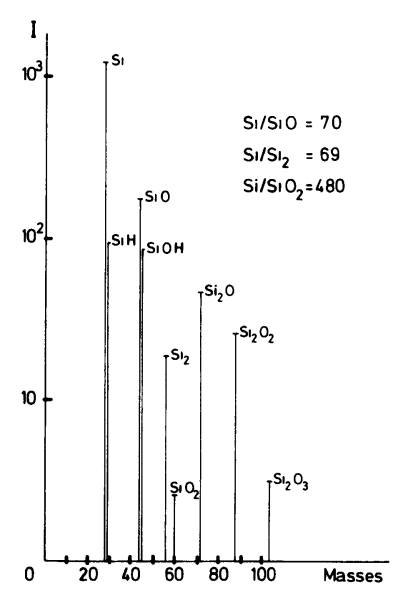

b)
Fig. 17. - a) Groupements polyatomiques du quartz $\mathrm{SiO}_{2}-$ ions primaires $\mathrm{A}^{+}$; intensité exprimée en unité relative. Mesures de Mme Havette [31]. b) Groupements polyatomiques de la couche oxydée-ions primaires $\mathrm{A}^{+}$; intensité exprimée en unité relative. Nos mesures.

[a) Polyatomic ions of the quartz $\mathrm{SiO}_{2}$-primary ions $\mathrm{A}^{+}$; intensity expressed in relative units. Results of Mme Havette [31]. b) Polyatomic ions of the oxide layerprimary ions $\mathrm{A}^{+}$; intensity expressed in relative units. This work.]

masses $M_{i}$ et $M_{j}$, le rapport mesuré $R_{i j}$ (rapport des ions comptés pour les masses $i$ et $j$ ) est comparé au rapport standard d'abondance isotopique tabulé $R_{i j}$ $\left({ }^{28} \mathrm{Si}=92,2 \%,{ }^{29} \mathrm{Si}=4,7 \%,{ }^{30} \mathrm{Si}=3,09 \%\right)$. Les écarts relatifs ou discriminations $\Delta_{i j}$ définis par 


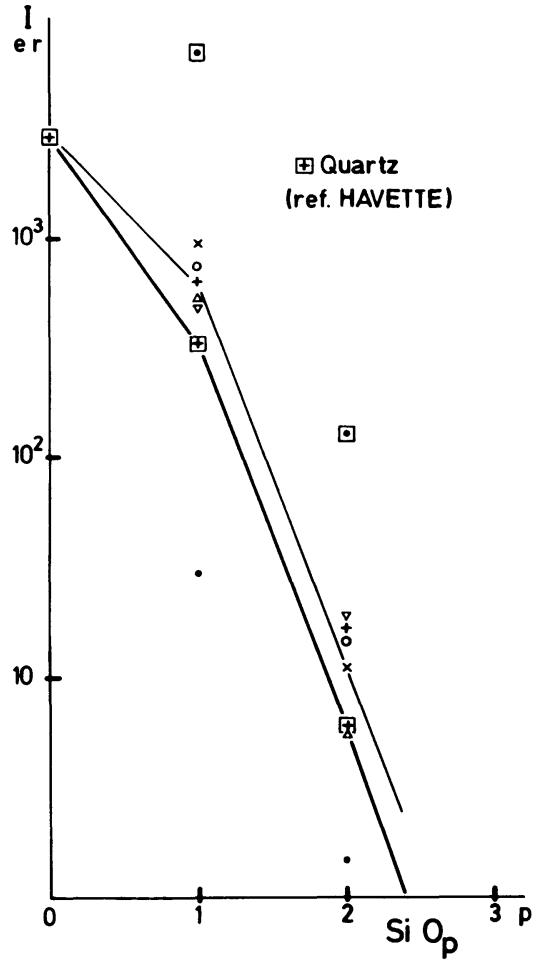

a)

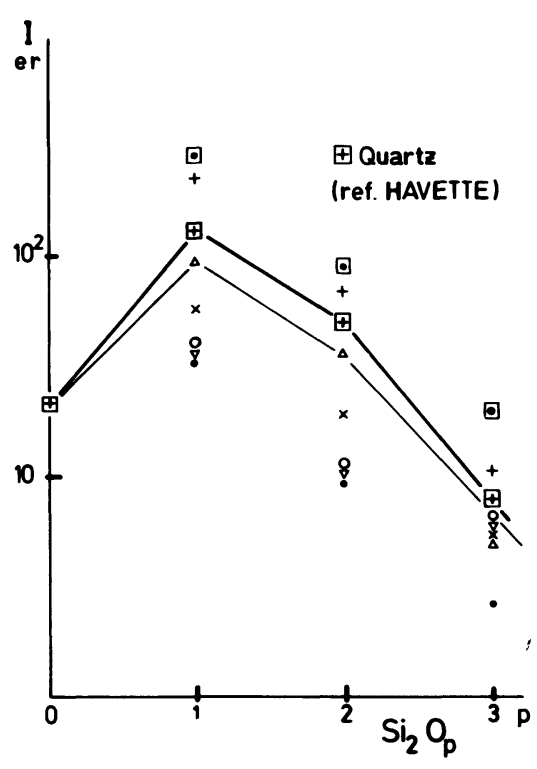

b)

Fig. 18. - Emission comparative des différents ions moléculaires se rapportant à $\mathrm{Si}$ (intensité exprimée en unités relatives) : a) $\left.\mathrm{SiO}_{p} ; b\right) \mathrm{Si}_{2} \mathrm{O}_{p}$. $⿴ 囗 十$ Mesures de Mme Havettequartz $\mathrm{SiO}_{2} ; \square$ Premières couches pulvérisées (durée d'attaque de 0 à $\left.200^{\prime \prime}\right) ;+\triangle \times O \nabla:$ balayages intermédiaires dans l'épaisseur de la couche oxydée, $\bullet$ dernières couches pulvérisées (durée d'attaque de 1250 à 1450").

[Comparative emission yields of the different molecular ions of : a) $\left.\mathrm{SiO}_{p} ; b\right) \mathrm{Si}_{2} \mathrm{O}_{p}$. $⿴ 囗 十$ Results of Mme Havettequartz $\mathrm{SiO}_{2} ; \square$ Topmost sputtered layers (sputtering time : $\left.0-200^{\prime \prime}\right) ;+\triangle \times O \nabla:$ indepth scans into the oxide layer, $\bullet$ dephest sputtered layers (sputtering time : 1250 $\left.1450^{\prime \prime}\right)$.]

REVUE DE PHYSIQUE APPLIQUÉE. — T. 17, № 10, OCTOBRE 1982
$\Delta_{i j}=\left[\left(R_{i j} / R_{i j}^{0}\right)-1\right]$ varient avec la composition de la matrice dans laquelle sont mesurés les $R_{i j}$. Les rapports ${ }^{29} \mathrm{Si} /{ }^{28} \mathrm{Si}$ et ${ }^{30} \mathrm{Si} /{ }^{28} \mathrm{Si}$ ont été mesurés sur les spectres successifs. Les écarts $\Delta{ }^{29 / 28} \mathrm{Si}$ et $\Delta{ }^{30 / 28} \mathrm{Si}$ varient dans des limites importantes pour des durées d'attaque qui correspondent à la pulvérisation de la couche d'oxyde puis deviennent constants et voisins de -4 et $5 \%$ lorsque l'érosion se fait dans le substrat de composition constante. L'accord entre l'évolution des rapports isotopiques et la variation de la composition de la matrice est contrôlé facilement sur les spectres où sont enregistrées simultanément les différentes masses.

4.3 CORRÉlation COUlEurs-ORIENTATIONS CRISTALLINES. - L'étude a porté sur des échantillons prélevés en différentes zones de 3 lingots (élaboration C.G.E. : $\mathrm{E}_{\mathrm{I}}, \mathrm{E}_{\mathrm{V}_{1 \mathrm{a} 4}}$ et $\mathrm{E}_{\mathrm{V}_{11 \mathrm{a} 3}}$ ). L'orientation cristalline est déterminée à l'aide des diagrammes de Laue. Dans le tableau VII sont reportés les résultats obtenus sur la plaquette $E_{1}$ pour différents grains repérés sur la figure 19; voir la photo couleur 3 de la surface après attaque électrochimique. La surface anodique $(\varnothing=20 \mathrm{~mm})$ recouvre les zones 1 à 5 étudiées au paragraphe 3.3. La densité réticulaire est corrigée en tenant compte des faibles désorientations : $\tau, \sigma$ et $\rho$ des plans $(h k l)$ par rapport au plan de la surface attaquée.

Dans la dernière colonne sont reportées les mesures d'épaisseur qui confirment les observations optiques : épaisseur totale de la couche en plusieurs points du pourtour de l'anode et variations d'épaisseur entre grains adjacents de coloration différente.

Les résultats reportés dans les tableaux VI et VII indiquent toujours un très bon accord entre variations d'épaisseur mesurées et changements de couleur. L'existence d'une corrélation reproductible entre couleurs et orientations est vérifiée. D'après les mesures de l'épaisseur totale du dépôt, en différents points, la couche intermédiaire reste indépendante de l'orientation cristalline; elle est de l'ordre de $13700 \pm 300 \AA$. Par contre, l'épaisseur de la couche d'oxyde dépend de la densité réticulaire des plans attaqués. Une relation simple a été établie entre cette densité $d_{\mathrm{r}}$ et l'épaisseur $l$

$l$ exprimée en $\AA$,

$$
l=\mathrm{e}^{\left(a d_{r}+b\right)}
$$

$d_{\mathrm{r}}$ exprimée en atome. $\AA^{-2}$.

Les paramètres $a$ et $b$ sont constants. Avec l'échantillon $\mathrm{E}_{1}$ pour les conditions électrolytiques adoptées :

$$
\left\{\begin{array}{l}
a \cong 10,7 \pm 0,2 \\
b \cong 7,2 \pm 0,1
\end{array}\right.
$$

Les grains sont planimétrés et une distribution relative des orientations cristallines est obtenue très rapidement d'après les surfaces de couleur donnée. Pour la zone représentée, photo 3 , la distribution est la suivante : totale

- coloration jaune plan (111) $17,3 \%$ de la surface 
Tableau VII. - Corrélation couleur. Densité réticulaire

[Correlation between colour and reticular density.]

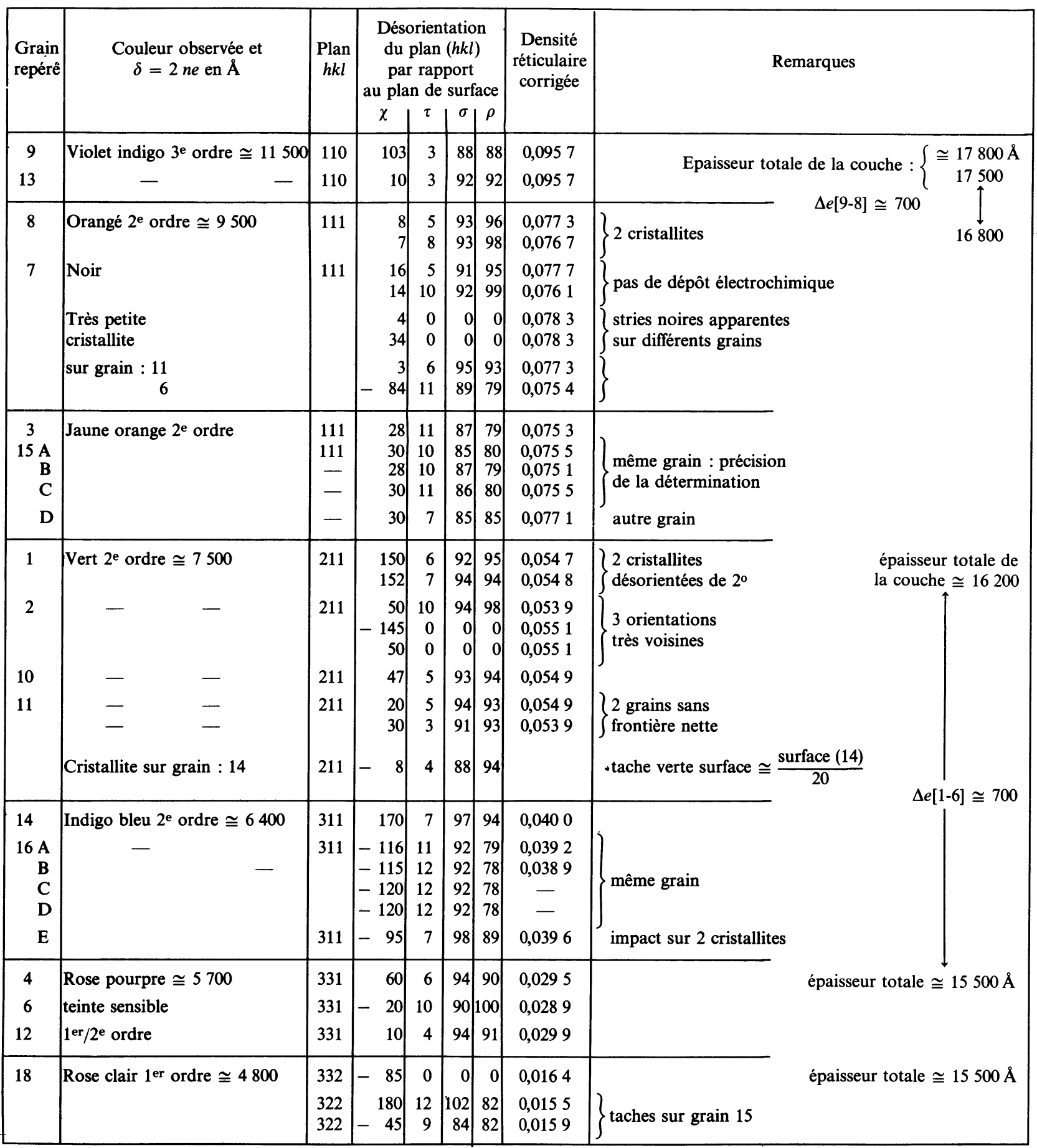

- coloration verte plan (211) $25,2 \%$ de la surface totale - coloration indigo plan (311) $16,1 \%$ de la surface totale

- coloration mauve-rosé plan (331) $22,0 \%$ de la surface totale.

soit environ $81 \%$ de la surface totale.

Les résultats obtenus sur les autres échantillons ne sont pas reportés ici. Sur toutes les plaquettes étudiées 50 à $60 \%$ de la surface sont représentés par 3 couleurs bien définies correspondant à 3 orientations différentes (voir Tableau VIII). Une corrélation couleur-orientation existe toujours reproductible mais avec un décalage des couleurs de lame mince; les paramètres $a$ et $b$ varient avec la vitesse de formation de la couche qui dépend de la concentration en impuretés du silicium polycristallin.

Les taux d'impuretés, autres que les éléments 


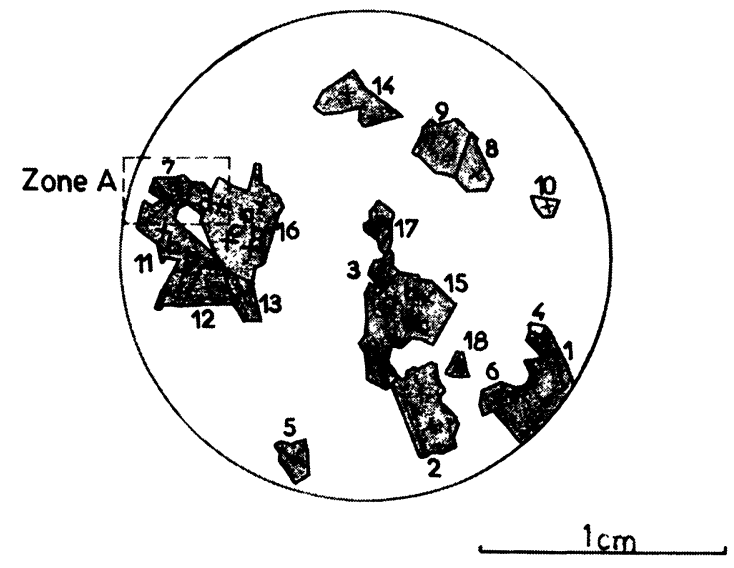

a)

Fig. 19. - Numérotation des grains pour lesquels l'orientation cristalline a été mesurée : a) Surface complète de l'attaque $\varnothing=20 \mathrm{~mm} ; b$ ) Zone A entourant le grain 7 très maclé.

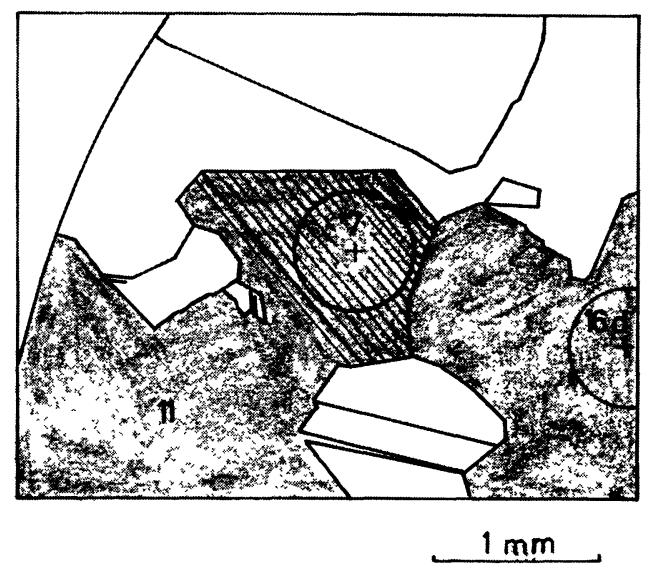

b)

[Numbering of the observed grains :a) Total etched surface $\varnothing=20 \mathrm{~mm} ; b$ ) Zone $\mathrm{A}$ around the grain $\mathrm{n}^{\circ} 7$.]

Tableau VIII. - Répartition en surface des plans d'orientation donnée

[Distribution of the planes with a defined orientation on the wafer surface.]

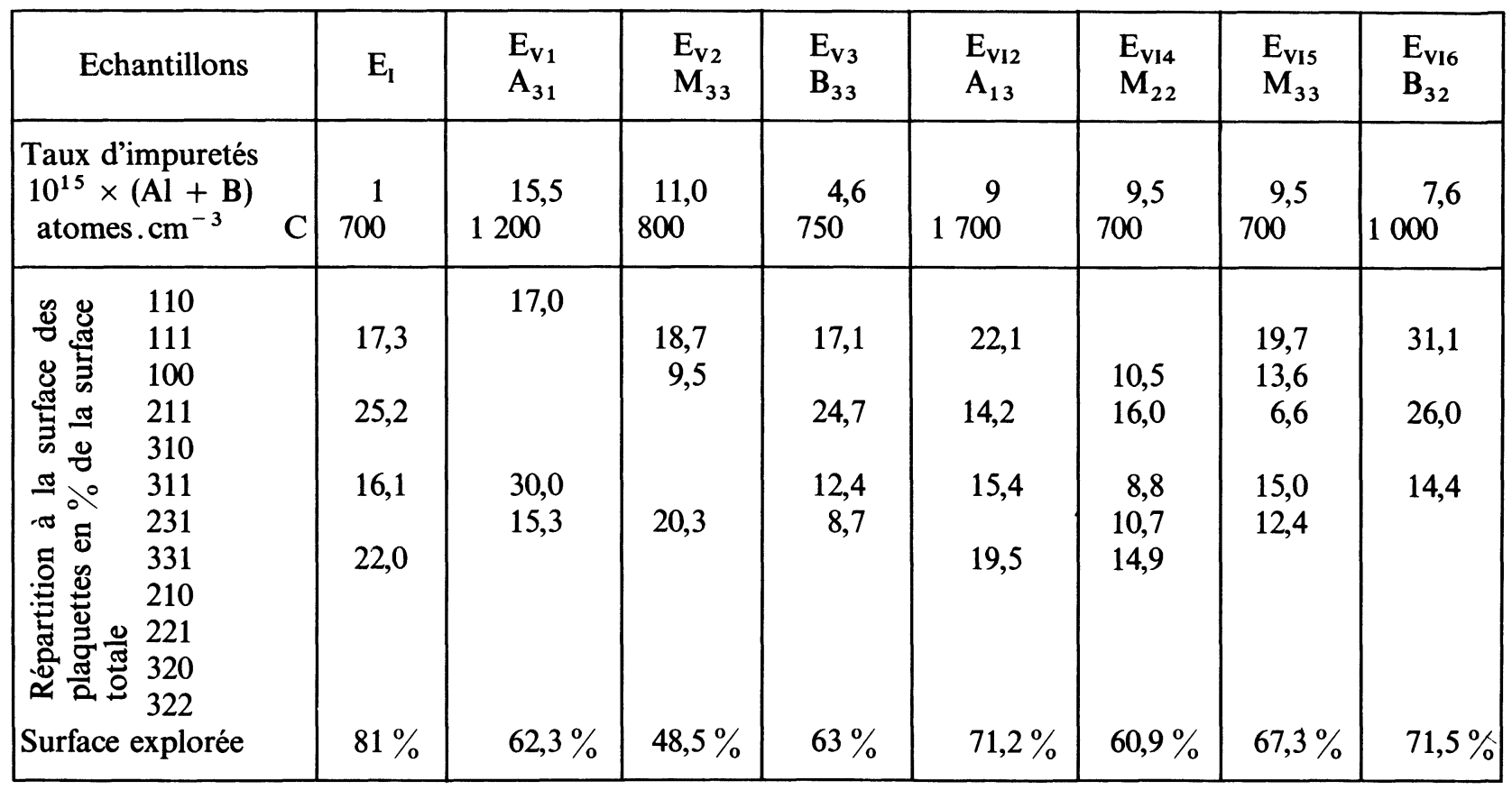

dopants incorporés tels que bore et aluminium et l'importante pollution de carbone due au creuset, restent $<10^{15}$ atomes $/ \mathrm{cm}^{3}$. Les analyses, faites par spectrométrie de masse à étincelle, spectrométrie IR et par activation protonique et neutronique, ne sont pas reportées dans le tableau I.

L'étude du comportement chimique et électrique des joints de grains dans le silicium polycristallin [33]. a mis en évidence une ségrégation importante du carbone dans ces joints. Il a été indiqué précédemment qu'une partie du carbone se retrouve également sous forme de graphite hexagonal et du carbure de silicium à l'état de cristallites isolés. Une dissolution uniforme du carbone dans les grains du silicium polycristallin n'est pas retenue ici.

Les variations d'épaisseur du dépôt électrolytique, mesurées et contrôlées à partir du décalage des couleurs observées sur les différents échantillons, ont été reliées à la variation du taux global des impuretés dopantes B et $\mathrm{Al}$.

L'échantillon $\mathrm{E}_{1}$ contient le plus faible taux d'impuretés. Dans les autres, la concentration totale des 
dopants $(\mathrm{B}+\mathrm{Al})$ varie de 4 à $15,5 \times 10^{15}$ atomes $/ \mathrm{cm}^{3}$. Tant que cette concentration reste inférieure à $9 \times 10^{15}$ atomes $/ \mathrm{cm}^{3}$, la variation de la vitesse de formation de la couche $\Delta e / \Delta t$ en fonction de la variation de concentration $\Delta c$ reste linéaire. Cette linéarité n'est plus observée sur les échantillons $E_{V_{1 \text { et2 }} \text {; la }}$ vitesse de formation de la couche augmente plus rapidement.

De plus, la répartition des orientations cristallines varie. Les directions de croissance [110] et [100] parallèles à l'axe d'étirage sont fortement défavorisées tant que les taux de dopage $(\mathrm{Al}+\mathrm{B})$ restent $<9 \times 10^{5}$ atomes $/ \mathrm{cm}^{3}$. Puis la surface des plaquettes occupées par les plans (110) et (100) augmente avec la concentration des impuretés.

Les résultats sont encore trop fragmentaires pour confirmer une relation entre orientations préférentielles et taux de dopage.

Remarques sur la limite de la méthode électrolytique. - a) Etalonnage. - Expérimentalement, il est possible de contrôler les paramètres de l'électrolyse et de les rendre reproductibles. Par contre, les ségrégations d'impuretés inhomogènes dans la masse (sommet et périphéries du lingot, joints de grains), la contamination du matériau au cours de son élaboration sont autant de paramètres difficilement contrôlables qui nécessitent un réétalonnage rapide pour chaque plaquette taillée en différentes régions de lingots obtenus à partir d'élaborations variées. Pour cela après chaque électrolyse, il suffit de déterminer les orientations cristallines de quelques grains. Les dimensions de la cuve d'électrolyse peuvent être augmentées afin que l'anode recouvre une section complète du lingot. Le dépouillement d'une dizaine de diagrammes de Laue obtenus par réflexion du faisceau de rayons $X$ sur quelques grains correctement choisis, permettra d'obtenir rapidement une carte des orientations cristallines à la surface des cellules.

b) Variation de teinte sur un même grain. - Ex. grain $\mathrm{n}^{\circ} 15$, figure $19 a$. On vérifie que le faible changement de couleurs ne provient pas d'une déformation des plans réflecteurs ni d'une variation du taux d'impureté dans un même grain de grande taille. L'inclusion de petits cristallites d'orientations différentes peut intervenir. De même une inhomogénéité de l'attaque électrochimique peut provoquer ces variations. Un contrôle précis de la géométrie du montage et de la concentration de l'électrolyte en tout point de la surface anodique évite ce défaut. De même le développement des photographies couleurs doit être surveillé avec soin. c) Grains non colorés. - Ex. grain no 7, figure $19 b$. Les stries noires serrées observées sur ces grains représentent les traces de plans de macle | plans (111) | sur la surface de l'électrode. Après attaque, aucune coloration n'apparaît; ce qui signifie que le dépôt électrolytique ne s'est pas formé; la concentration " électrons-trous » à l'interface silicium-électrolyte étant trop faible. Il faut admettre que les dislocations intrinsèques aux plans de macles ou des dislocations extrinsèques de densité élevée diminuent la mobilité des porteurs. Ceci est confirmé par les mesures en E.B.I.C. où ces grains apparaissent toujours inactifs.

5. Conclusion. - Différentes techniques, généralement utilisées pour définir les orientations cristallines ont été appliquées au silicium polycristallin massif. Une méthode nouvelle, basée sur l'attaque électrochimique avec étalonnage de l'épaisseur du dépôt d'oxyde formé en fonction de la densité réticulaire du plan de surface attaqué, a été développée; sa précision et sa reproductibilité ont été déterminées. Bien que son imprécision soit importante tant dans l'évaluation des surfaces occupées par les plans d'indices donnés (à $\pm 10 \%$ près) que dans la détermination des orientations des plans $\left(\grave{a} \pm 8^{\circ}\right)$, cette méthode a été retenue pour un contrôle rapide d'élaboration industrielle d'un silicium polycristallin massif.

Des orientations de croissance cristalline privilégiées, ont été mises en évidence. Elles dépendent des gradients de température au cours du tirage du silicium et du taux d'impuretés présentes. Des zones de cristallinités différentes apparaissent dans le lingot. Des études de texture de solidification sont en cours sur ce matériau. Elles doivent permettre de corréler orientations cristallines et propriétés photovoltaïques

Ce travail a été réalisé aux Laboratoires de Recherche de la Compagnie Générale d'Electricité à Marcoussis au cours d'un stage « relation C.N.R.S.Industrie ». Je remercie M. Dumas, Directeur de la division Matériaux et M. Barraud, Responsable du Groupe « analyses » pour l'aide matérielle qu'ils m'ont apportée. L'élaboration du silicium massif polycristallin a été mise au point par Mrs. Fally et Guenel à la C.G.E. Les conditions optimales de l'attaque électrolytique ont été définies par M. Tourillon, chercheur C.N.R.S., au cours d'un stage industriel effectué dans le même laboratoire. Je remercie également les chercheurs du Laboratoire de Physique des Solides d'Orsay, de l'Institut Laue-Langevin et du Centre de Recherche de Bellevue, qui ont facilité ma tâche en mettant à ma disposition différents montages de diffraction des rayons $\mathrm{X}$, des neutrons ainsi que l'analyseur ionique.

\section{Bibliographie}

[1] Proceedings of the International Conference on Photovoltaic Solar Energy (Berlin, Avril 1979, Cannes, Octobre 1980, Orlando, mai 1981).
[2] Garone, L. G., Hari Rao, C. V., Morrison, A. D., Surek, T. and Ravi, K. V., Appl. Phys. Lett. 29 (1976) 511. 
[3] Schwuttke, G. H., Ciszek, T. F., Yang, K. H. and Kran, A., I.B.M. J. Res. Develop. 22 no 4 (July 1978).

[4] Belouet, C., Brissot, J. J. et Martres, R., Proc. Col. Int. sur l'électricité solaire (Toulouse) 1976, p. 191.

Belouet, C., Schneider, J., Belin, C. and Martres, R., XIVth IEEE SPSC (San Diego) 1980, p. 49.

[5] Zook, J. D., Schuldt, S. B., MaCIOLEK, R. B. and Heaps, J. D., Proc. of the XIIth IEEE SPSC (Washington) 1978, p. 472.

[6] Fischer, H. and Pschunder, W., IEEE Trans. Electron Devices ED-24 (1977) 483.

FISCHER, H., Festkörperprobleme XVIII (1978) 19-32.

[7] CiszeK, T. F., Schwuttke, G. H. and Yang, K. H., J. Crystal Growth 46 (1979) 527-533; I.B.M. J. Res. Develop. 23 no 3 (May 1979).

[8] Fally, J. and Guenel, C., Proc. of the Int. Conf. on Photovoltaic Solar Energy, Cannes France, 1980 , p. 598-602.

[9] Roy, K., Rasch, K. D. and Fischer, H., XIVth IEEE SPSC (San Diego) 1980, p. 897.

[10] Legros, B., David, B. R., Boissy, M. C., Lebailly, J., Ngo, T. P. et Fabre, E., Proc. of the Int. Conf. on Photovoltaic Solar Energy, Cannes France, octobre 1980, p. 593-597.

[11] Greninger, A. D., Z. Kristallogr. 91 (1935) 424; Trans. AIME 117 (1935) 75 et 122 (1936) 74.

[12] GuInIER, A., Théorie et Technique de la radiocristallographie (Dunod) 1956.

[13] Henry, N. F., Lipson, H. and Wooster, W. A., The interpretation of $X$-ray diffraction photographs (MacMillan, London) 1960.

[14] Ном, T., Kiszenick, W. and Post, B., J. Appl. Crystallogr. 8 (1978) 457-458.

[15] TURNER, D. R., The surface chemistry of metals and semiconductors, Gatos Editor (J. Wiley and Sons, N.Y.) 1960 , p. 285-310.

[16] Memming, R. and Schwandt, G., Surf. Sci. 4 (1966) 104-124.
[17] Theunissen, M. J. J., J. Electrochem. Soc. 119 (1972) 351-360.

[18] Watanabe, Y., Arita, Y., Yokoyama, T. and IgaRASHI, Y., J. Electrochem. Soc. 122 no 10 (1975) 1351-1355.

[19] Arita, Y. and Sunohara, Y., J. Electrochem. Soc. 124 (1977) 285-295.

[20] Tourillon, G., Andonov, P., Godard, A. M. and BarRaud, J. Y., Journées d'électrochimie, Bruxelles $81,2-5$ juin.

[21] Tourillon, G., Andonov, P. and Barraud, J. Y., 161st Meeting of the Electrochemical Society, Montréal, Canada, May 9-14 1982.

[22] Tourillon, G., Andonov, P., Godard, A. M. and BARRAUd, J. Y., Soumis à Solar Cells.

[23] Foll, H., J. Electrochem. Soc. 127 no 9 (1980) 19251931.

[24] Nouveau traité de chimie minérale, P. PASCAL, Tome VIII, 2e fascicule (Masson Edition) 1965.

[25] Brodsky, M. H., Title, R. S., Weiser, K. and Pettit, G. D., Phys. Rev. 1 no 6 (March 1960).

[26] Salzberg, C. D. and Villa, J. J., J. Opt. Soc. Am. 47 (1957) 244.

[27] Bruhat, G., Cours de Physique Générale. Optique, 6e édition complétée par A. KASTLER (Masson édition) 1965.

[28] Mosseri, R., Thèse 3e cycle, Paris-VII (1979).

[29] Warren, B. E., X-ray diffraction (Addison Wesley Publishing Company) 1969.

[30] Watson, Gundu Rao, Ramaswany, Proc. R. Soc. A 143 (1934) 558.

[31] Havette-Ledebt, A., Thèse 3e cycle, Pétrographie, Orsay (1974).

[32] Slodzian, G., Lorin, J. C. et Havette, A., C.R. Hebd. Séan. Acad. Sci. Paris 291 B (29 sept. 1980) 121-124.

[33] Aucouturier, M., Rallon, O., Auco, M., Hervo, C., Mautref, M. et Belouet, C., Communications aux Journées d'Automne de la S.F.M., Paris, octobre 1981. A paraître dans les Mémoires Scientifiques de la Revue de Métallurgie. 